hep-ph/0304130

\title{
Neutrino Bilarge Mixing and Flavor Physics in the Flipped SU(5) Model
}

\author{
Chao-Shang Huang ${ }^{a}$, Tianjun $\mathrm{Li}^{b}$ and Wei Liao ${ }^{c}$ \\ ${ }^{a}$ Institute of Theoretical Physics, Academia Sinica, P. O. Box 2735, \\ Beijing 100080, China \\ ${ }^{b}$ School of Natural Science, Institute for Advanced Study, \\ Einstein Drive, Princeton, NJ 08540, USA \\ ${ }^{c}$ The Abdus Salam International Center for Theoretical Physics, \\ Strada Costiera 11, 34014 Trieste, Italy
}

\begin{abstract}
We have constructed a specific supersymmetric flipped SU(5) GUT model in which bilarge neutrino mixing is incorporated. Because the up-type and down-type quarks in the model are flipped in the representations ten and five with respect to the usual $\mathrm{SU}(5)$, the radiatively generated flavor mixing in squark mass matrices due to the large neutrino mixing has a pattern different from those in the conventional $\mathrm{SU}(5)$ and $\mathrm{SO}(10)$ supersymmetric GUTs. This leads to phenomenological consequences quite different from $\mathrm{SU}(5)$ or $\mathrm{SO}(10)$ supersymmetric GUT models. That is, it has almost no impact on $\mathrm{B}$ physics. On the contrary, the model has effects in top and charm physics as well as lepton physics. In particular, it gives promising prediction on the mass difference, $\Delta M_{D}$, of the $D-\bar{D}$ mixing which for some ranges of the parameter space with large $\tan \beta$ can be at the order of $10^{9} \hbar s^{-1}$, one order of magnitude smaller than the experimental upper bound. In some regions of the parameter space $\Delta M_{D}$ can saturate the present bound. For these ranges of parameter space, $t \rightarrow u, c+h^{0}$ can reach $10^{-5}-10^{-6}$ which would be observed at the LHC and future $\gamma-\gamma$ colliders.
\end{abstract}

\section{Introduction}

In recent years great progresses have been made on the flavor physics. Atmospheric neutrino [1] and solar neutrino [2] experiments together with the reactor neutrino [3, 4] experiments have established the oscillation solution to the solar and atmospheric neutrino anomalies [5, 6, 7]. The solution tells us that neutrinos have masses and mix with themselves in the propagations, i.e., they oscillate $[8,9,10]$. The recent result from the super-K collaboration and the combined analysis on the solar neutrino experiment result and the KamLAND experiment result give the best fit points $[5,6]$

$$
\begin{aligned}
& \Delta m_{23}^{2}=2.5 \times 10^{-3} \mathrm{eV}^{2}, \quad \sin ^{2} 2 \theta_{23}=1.0 \\
& \Delta m_{12}^{2}=7.3 \times 10^{-5} \mathrm{eV}, \quad \tan ^{2} \theta_{12}=0.41 ;
\end{aligned}
$$

where $\Delta m_{i j}^{2}=m_{\nu_{i}}^{2}-m_{\nu_{j}}^{2}$ is the mass squared differences of the neutrinos in the mass eigenstates (possible signs neglected here), and $\theta_{i j}$ are the two-neutrino mixing angles. $\theta_{12}$ is for the solar neutrino oscillation and $\theta_{23}$ is for the atmospheric neutrino oscillation. Moreover, the CHOOZ 
experiment made a constraint on the $\theta_{13}$ for the mass differences observed in the atmospheric and solar neutrino experiments [4]

$$
\left|\sin \theta_{13}\right| \lesssim 0.16
$$

Understanding these masses and mixings is a challenge. The smallness of the masses can be understood via the see-saw mechanism [11]. Namely, the heavy right-handed Majorana neutrinos, whose masses violate the lepton number symmetry, simply decouple in the low energy physics and give very small lepton number violating effects in the low energy phenomena, which are the extremely small Majorana masses of the left-handed neutrinos. The present experiments allow three typical solutions to the neutrino masses: the degenerate case with $m_{1} \sim m_{2} \sim m_{3} \sim 10^{-1} \mathrm{eV}$; hierarchy case with $m_{1} \ll m_{2} \approx \sqrt{\Delta m_{21}^{2}}, m_{2} \ll m_{3} \approx \sqrt{\Delta m_{32}^{2}}$; inverse hierarchy case with $m_{3} \ll m_{1} \sim m_{2} \sim \sqrt{\Delta m_{23}^{2}}$.

In view of the beautiful picture raised by Weinberg-Wilczek-Zee-Fritsch (WWZF) [12] that the small quark mixing in the CKM matrix is related to the large quark mass hierarchy, people feel challenged a lot by the presence of the neutrino bilarge mixing. Some people worried that we should have degenerate mass matrix to understand it. However, in the WWZF scenario only the symmetric mass matrix is used. If allowing asymmetric form for the mass matrix which for example may well be generated by the elegant Froggatt-Nielsen (FN) mechanism [13], we can accomodate the large mass hierarchy with large mixings [14]. If one works with an effective theory, e.g., the minimal supersymmetric Standard Model together with right-hand neutrinos $(\mathrm{MSSM}+\mathrm{N})$, at a low energy scale (say, the electro-weak scale), one can content oneself by using the WWZF scenario to understand the smallness of quark mixing, and the see-saw and FN mechanism to understand the largeness of neutrino mixing. However, if one works with a theory, e.g., a grand unification theory (GUT), in which quarks and leptons are in a GUT multiplet, one has to answer: can we explain simultaneously the smallness of quark mixing and the largeness of neutrino mixing in the theory? If we can, then what are the phenomenological consequences in the theory? There are several recent works to tackle these problems in SU(5) or $\mathrm{SO}(10)$ GUTs $[15,16,17,18,19,20]$.

As known for a long time in the framework of the supersymmetric see-saw mechanism, we are able to predict the lepton flavor violating (LFV) effects [21, 22]. The flavor structure in the neutrino Yukawa couplings can be transformed to the soft SUSY breaking masses of the left-handed sleptons which then give implications on the $\mu \rightarrow e \gamma$ and $\tau \rightarrow \mu, e+\cdots$ processes. In the models of the grand unification theory, e.g., in $S U(5)$ or $S O(10)$, quark fields are unified with leptons in the representations of GUT gauge group, and from the lepton Yukawa couplings we are able to get the flavor mixings in down-type squark mass matrix $[23,15,17,20]$. These flavor mixings are something beyond those described by the CKM mixing and can give the interesting phenomenological implications for the Kaon and B meson physics. This approach has recently been used in SUSY SU(5) and SO(10) GUTs which incorporate the bilarge neutrino mixing and can give the significant phenomenological predictions if the bilarge neutrino mixing is from the lepton Yukawa couplings $[15,17,20]$.

In the present paper, we address the problems in the supersymmetric flipped $S U(5)$ model. As we know, the flipped $S U(5)$ unification model has several advantages: (i) $S U(5) \times U(1)$ is the minimal unified gauge group which provides neutrino masses; (ii) without high dimension Higgs representations; (iii) the natural splitting of the doublet and triplet components of the Higgs pentaplets and consequently the natural avoidance of dangerous dimension-5 proton 
decay operators; and (iv) the natural appearance of a see-saw mechanism for neutrino masses. Furthermore, in the context of 4-dimensional free fermionic string model-building, at level one $(k=1)$ of the Kac-Moody algebras [24], one can only obtain the Standard-like Model [25], the Pati-Salam Model [26] and the flipped $S U(5)$ model [27], because the dimensions of Higgs fields in the spectra are smaller than that of the adjoint representation. Of these, only flipped $S U(5)$ actually unifies the non-abelian gauge groups of the Standard Model (SM). And a proliferation of $U(1)$ factor is the norm.

We show that the fermion mass hierarchies and the mixings of quarks and leptons can all be well accommodated in the flipped $S U(5)$ model. Moreover, because in flipped $S U(5)$ the up-type and down-typ quarks are flipped in the representations ten and five with respect to the usual $\mathrm{SU}(5)$, the one loop radiative corrections to sfermion masses have a pattern different from those in $\mathrm{SU}(5)$ or $\mathrm{SO}(10)$ and consequently different phenomenological implications. We find that in flipped $S U(5)$ new effects appear in top and charm physics, in addition to lepton physics, and no effects in B physics, which is a novel feature quite different from the usual $\mathrm{SU}(5)$ and $\mathrm{SO}(10)$ GUTs and can be tested by incoming experiments.

The paper is organized as follows. In section II, after a brief review of the flipped $S U(5)$ model, we construct a specific model in which the bilarge mixing can be accomodated in the Yukawa couplings as well as the fermion mass hierarchy and the quark mixing. We will then discuss the radiatively corrected SUSY breaking soft terms with universal SUSY breaking at the Planck scale. In section III, we discuss the phenomenological consequences of our model. Section IV is devoted to conclusions and discussions. Finally, some conventions in MSSM+N are given in Appendix A, and the renormalization group equations (RGEs) in MSSM+N and flipped $\mathrm{SU}(5)$ are given in the Appendices B and C, respectively. Throughout the paper we assume no $C P$ violating phases appearing the leptonic sector to simplify the discussion.

\section{Flipped SU(5) Model and the Flavor Structure}

\subsection{The Flipped $S U(5)$ Model}

In this subsection, we would like to briefly review the Flipped $S U(5)[29,30]$ and construct a specific flipped $\mathrm{SU}(5)$ model. The gauge group for flipped $S U(5)$ model is $S U(5) \times U(1)_{X}$, which can be embedded in $S O(10)$ model. We can define the generator $U(1)_{Y^{\prime}}$ in $S U(5)$ as

$$
T_{\mathrm{U}(1)_{\mathrm{Y}^{\prime}}}=\operatorname{diag}\left(-\frac{1}{3},-\frac{1}{3},-\frac{1}{3}, \frac{1}{2}, \frac{1}{2}\right) .
$$

The hypercharge is given by

$$
Q_{Y}=\frac{1}{5}\left(Q_{X}-Q_{Y^{\prime}}\right)
$$

There are three families of fermions with the following $S U(5) \times U(1)_{X}$ transformation proporties

$$
F_{i}=(10,1), \bar{f}_{i}=(\overline{5},-3), l_{i}^{c}=(1,5),
$$

where $i=1,2,3$. As an example, the particle assignments for the first family are

$$
F_{1}=\left(Q_{1}, D_{1}^{c}, N_{1}\right), \bar{f}_{1}=\left(U_{1}^{c}, L_{1}\right), l_{1}^{c}=E_{1}^{c} .
$$


$Q$ and $L$ are the superfields of the quark and lepton doublets, $U^{c}, D^{c}, E^{c}$ and $N$ are the $C P$ conjugated superfields for the right-handed up-type quark, down-type quark, lepton and neutrino.

To break the GUT and electroweak symmetries, we introduce two pairs of Higgs representations

$$
H=(10,1), \bar{H}=(\overline{10},-1), h=(5,-2), \bar{h}=(\overline{5},+2) .
$$

We label the states in the $H$ multiplet by the same symbols as in the $F$ multiplet, and for $\bar{H}$ we just add "bar" above the fields. Explicitly, the Higgs particles are

$$
\begin{gathered}
H=\left(Q_{H}, D_{H}^{c}, N_{H}\right), \bar{H}=\left(\bar{Q}_{\bar{H}}, \bar{D}_{\bar{H}}^{c}, \bar{N}_{\bar{H}}\right), \\
h=\left(D_{h}, D_{h}, D_{h}, H_{1}\right), \bar{h}=\left(\bar{D}_{\bar{h}}, \bar{D}_{\bar{h}}, \bar{D}_{\bar{h}}, H_{2}\right) .
\end{gathered}
$$

We also add one singlet $S$.

To break the $S U(5) \times U(1)_{X}$ gauge symmetry down to the SM gauge symmetry, we introduce the GUT superpotential

$$
W_{\mathrm{GUT}}=\lambda_{1} H H h+\lambda_{2} \bar{H} \bar{H} \bar{h}+S\left(\bar{H} H-M_{\mathrm{V}}^{2}\right) .
$$

There is only one F-flat and D-flat direction, which can always be rotated along the $N_{H}$ and $\bar{N}_{\bar{H}}$ directions. So, we obtain that $\left.\left\langle N_{H}\right\rangle=<\bar{N}_{\bar{H}}\right\rangle=M_{\mathrm{V}}$. In addition, the superfields $H$ and $\bar{H}$ are eaten or acquire large masses via the supersymmetric Higgs mechanism, except for $D_{H}^{c}$ and $\bar{D}_{\bar{H}}^{c}$. And the superpotential $\lambda_{1} H H h$ and $\lambda_{2} \bar{H} \bar{H} \bar{h}$ combine the $D_{H}^{c}$ and $\bar{D}_{\bar{H}}^{c}$ with the $D_{h}$ and $\bar{D}_{\bar{h}}$, respectively, to form the massive eigenstates with masses $\lambda_{1}<N_{H}>$ and $\lambda_{2}<\bar{N}_{\bar{H}}>$. So, we naturally have the doublet-triplet splitting due to the missing partner mechanism. Because the triplets in $h$ and $\bar{h}$ only have small mixing through the $\mu$ term, the higgsino-exchange mediated proton decay are negligible, i.e., we do not have dimension-5 proton decay problem. The singlet $S$ can have GUT scale mass and decouple in the low energy theory.

The SM fermion masses are from the following superpotential*

$$
W_{\text {Yukawa }}=\frac{1}{8} F_{i}\left(Y_{10}\right)_{i j} F_{j} h+F_{i}\left(Y_{\overline{5}}\right)_{i j} \bar{f}_{j} \bar{h}+l_{i}^{c}\left(Y_{1}\right)_{i j} \bar{f}_{j} h+\mu h \bar{h}+\frac{1}{2 M_{*}} F_{i}\left(Y_{R}\right)_{i j} F_{j} \bar{H} \bar{H}
$$

After the $S U(5) \times U(1)_{X}$ symmetry is broken down to the SM group, the superpotential gives

$$
\begin{aligned}
W_{S S M}= & D_{i}^{c}\left(Y_{10}\right)_{i j} Q_{j} H_{1}+U_{i}^{c}\left(Y_{\overline{5}}\right)_{j i} Q_{j} H_{2}+E_{i}^{c}\left(Y_{1}\right)_{i} L_{j} H_{1}+N_{i}\left(Y_{\overline{5}}\right)_{i j} L_{j} H_{2} \\
& \left.+\mu H_{1} H_{2}+\frac{1}{2}\left(M_{N}\right)_{i j} N_{i} N_{j}+\cdots \text { (decoupled below } M_{G U T}\right) .
\end{aligned}
$$

Thus, at the $M_{G U T}$ scale we have

$$
Y_{U}=Y_{\overline{5}}^{T}, \quad Y_{D}=Y_{10}, \quad Y_{N}=Y_{\overline{5}}, \quad Y_{E}=Y_{1}
$$

*In popular flipped SU(5) models one usually introduces more singlets and constructs a renormalizable superpotential. Here instead we introduce a non-renormalizable term to give the right-hand neutrino masses in order to avoid introducing more fields. 
The right-handed neutrino mass matrix $M_{N}$ is given by $Y_{R}$.

Assuming the right-handed neutrinos are heavy, they decouple at the low energy scale. After we integrate out the right-handed neutrinos, the left-handed neutrino Majorana masses are given by

$$
W_{\mathrm{m}_{\nu_{\mathrm{L}}}}=\frac{M_{*}}{M_{V}^{2}}\left(Y_{\overline{5}}^{T} Y_{R}^{-1} Y_{\overline{5}}\right)_{i j} L_{i} L_{j} H_{2} H_{2} .
$$

Thus, if $\left(Y_{R}\right)_{33} M_{V}^{2} / M_{*} \sim 10^{14} \mathrm{GeV}$, we obtain the correct $\tau$ neutrino $\left(\nu_{\tau}\right)$ mass implied by the atmospheric neutrino oscillation experiment because the $\tau$ neutrino $\left(\nu_{\tau}\right)$ Dirac mass is equal to the top quark mass at the GUT scale due to Eq. (13). In addition, the left-handed neutrino Majorana mass matrix, which is symmetric, can be taken to be arbitrary because the Majorana mass matrix $Y_{R}$ for the right-handed neutrinos is arbitrary.

We can see from Eq. (11) that the up-type quark Yukawa matrix is the transpose of the neutrino Dirac Yukawa matrix and the down-type quark Yukawa matrix is symmetric. Because in the superpotenyial $W_{S S M}$, the up-type quark mass matrix, the lepton mass matrix and the symmetric left-handed neutrino Majorana mass matrix are arbitrary, we can generate the correct SM fermion mass matrices, the CKM matrix and the neutrino mixing matrix, although the down-type quark mass matrix is symmetric.

To be concrete, we present a realistic sample for the SM fermion mass matrices in the Flipped $S U(5)$ model which accomodates the bilarge neutrino mixing. The discussion will be made without considering the $\mathrm{CP}$ violating phases, as noted in Introduction.

The ratios of the masses at the GUT scale [31] are

$$
\begin{aligned}
& m_{u}: m_{c}: m_{t} \sim \lambda^{7}: \lambda^{4}: 1, \\
& m_{d}: m_{s}: m_{b} \sim \lambda^{4}: \lambda^{2}: 1, \\
& m_{e}: m_{\mu}: m_{\tau} \sim \lambda^{5}: \lambda^{2}: 1 .
\end{aligned}
$$

where $\lambda=0.22$. And the CKM matrix is given by

$$
V_{\mathrm{CKM}} \sim\left(\begin{array}{ccc}
1 & \lambda & \lambda^{3} \\
-\lambda & 1 & \lambda^{2} \\
-\lambda^{3} & -\lambda^{2} & 1
\end{array}\right)
$$

In the hierarchy case, we can estimate the ratio of the neutrino masses

$$
m_{\nu_{2}}: m_{\nu_{3}} \sim \sqrt{\frac{\Delta m_{\text {solar }}^{2}}{\Delta m_{\text {atm. }}^{2}}} \sim \epsilon^{\prime}: 1,
$$

where $\epsilon^{\prime}$ is about 0.2 according to Eq. (1). The neutrino mixing matrix can be parametrized in the standard way

$$
U=\left(\begin{array}{ccc}
c_{12} c_{13} & s_{12} c_{13} & s_{13} e^{-i \delta_{13}} \\
-s_{12} c_{23}-c_{12} s_{23} s_{13} e^{i \delta_{13}} & c_{12} c_{23}-s_{12} s_{23} s_{13} e^{i \delta_{13}} & s_{23} c_{13} \\
s_{12} s_{23}-c_{12} c_{23} s_{13} e^{i \delta_{13}} & -c_{12} s_{23}-s_{12} c_{23} s_{13} e^{i \delta_{13}} & c_{23} c_{13}
\end{array}\right),
$$


where $c_{i j}=\cos \theta_{i j}, s_{i j}=\sin \theta_{i j}$, and $\delta_{13}$ is the $C P$ violating phase which is taken as zero as those Majorana phases in our discussion, as we noted in the Introduction. In view of Eq. (2), one has $c_{13} \gtrsim 0.98$, then, to a good approximation, one has

$$
U=\left(\begin{array}{ccc}
c_{12} & s_{12} & s_{13} \\
-\frac{s_{12}}{\sqrt{2}} & \frac{c_{12}}{\sqrt{2}} & \frac{1}{\sqrt{2}} \\
\frac{s_{12}}{\sqrt{2}} & -\frac{c_{12}}{\sqrt{2}} & \frac{1}{\sqrt{2}}
\end{array}\right)
$$

where $c_{23}=s_{23}=\frac{1}{\sqrt{2}}, c_{13}=1$, and $s_{12}<c_{12}$ with $\theta_{12} \sim 33^{0}$ are globally consistent with all neutrino experiments known so far.

The up-type quark Yukawa and the neutrino Dirac Yukawa couplings can take the following form

$$
Y_{U}=\left(Y_{\nu}\right)^{T} \sim y_{t}\left(\begin{array}{ccc}
\lambda^{7} & \lambda^{6} & \lambda^{4} \\
\lambda^{5} & \lambda^{4} & \lambda^{2} \\
\lambda^{3} & \lambda^{2} & 1
\end{array}\right),
$$

the down-type Yukawa coupling $Y_{D}$ takes the symmetric form

$$
Y_{D} \sim y_{b}\left(\begin{array}{ccc}
\lambda^{4} & 0 & 0 \\
0 & \lambda^{2} & 0 \\
0 & 0 & 1
\end{array}\right)
$$

and the lepton Yukawa coupling $Y_{E}$ can take the form

$$
Y_{E} \sim \frac{y_{\tau}}{\sqrt{2}}\left(\begin{array}{ccc}
\sqrt{2} a \lambda^{5} & \sqrt{2} b \lambda^{5} & \sqrt{2} \epsilon \lambda^{5} \\
-b \lambda^{2} & a \lambda^{2} & \lambda^{2} \\
b & -a & 1
\end{array}\right)
$$

where $a^{2}+b^{2}=1, \epsilon$ is smaller than one. In the base where the charged leptons are mass eigenstates and assuming that the same unitary matrix $U$ makes both the lepton mass matrix and the left-handed neutrino Majorana mass matrix diagonal, we obtain

$$
\sin \theta_{13} \approx \epsilon, \quad \tan \theta_{12} \approx \frac{b}{a}, \quad \tan \theta_{23}=1 .
$$

The bilarge neutrino mixing, Eq. (21), follows if $\epsilon \lesssim 0.16, b$ and $a$ are both of the order one and $b<a$. The prediction on $\theta_{13}$ heavily depends on the non-diagonal entries in the lefthanded neutrino Majorana mass matrix since we would like to get a small $\theta_{13}$. Because the right-handed neutrino mass matrix is arbitrary, as noted above, an appropriate form of the right-handed neutrino mass matrix can be taken to maintain the relation of $\theta_{13}$ in Eq. (25). The left-handed neutrino Majorana mass matrix, Eq. (14), is given for the flavor fields $\nu_{\alpha}$, $\alpha=e, \mu, \tau$. By the unitary transformation $U$ given in Eq. (21), we obtain the left-handed neutrino masses which can have mass hierarchy as

$$
m_{\nu_{1}}: m_{\nu_{2}}: m_{\nu_{3}} \sim \epsilon^{\prime \prime}: \epsilon^{\prime}: 1
$$

where the mass eigenvalues $m_{\nu_{i}}$ and consequently $\epsilon^{\prime \prime}$ and $\epsilon^{\prime}$ are determined by the matrix $\left(Y_{\overline{5}}^{T} Y_{R}^{-1} Y_{\overline{5}}\right)$ ( see Eq.(14)) with $Y_{\overline{5}}^{T}=Y_{U}$. In order to get $\epsilon^{\prime \prime}<\epsilon^{\prime} \sim \lambda$, the eigenvalues, $M_{N_{i}}$, of the right-handed neutrino Majorana mass matrix $M_{N}$ should have a hierarchy as large as

$$
M_{N_{2}}: M_{N_{3}} \sim \lambda^{7}: 1
$$


which for the $M_{N_{3}} \sim 10^{14-15} \mathrm{GeV}$ gives $M_{N_{2}} \sim 10^{9-10} \mathrm{GeV}$.

In the above discussions, the hierarchies in fermion mass spectra are obtained due to the hierarchies in Yukawa couplings. An elegant mechanism to understand this fermion mass hierarchy problem is the Froggatt-Nielsen mechanism [13]. However, the $U(1)$ global symmetry is not enough to generate the correct fermion masses, CKM matrix and neutrino mixing matrix. So, we need to consider the larger global symmetry, for instance, $S U(2) \times U(1)$ family symmetry. This is out of the scope of this paper and we will not consider it here.

Before proceeding, a remark is in place. The presence of the non-renormalizable operators might affect the predictions based on the renormalizable Yukawa couplings in Eq. (11). In our model, we do not have the dimension-5 non-renormalizable operators which can give the SM fermion masses except the operators which give the right-handed neutrino Majorana masses. There are dimension-6 non-renormalizable operators, for example,

$$
W_{\mathrm{np}}=\frac{1}{M_{*}^{2}} \lambda_{i j}^{3}\left[F_{i}(\bar{H} h)\right] F_{j} H+\frac{1}{M_{*}^{2}} \lambda_{i j}^{4}\left[F_{i} \bar{H}\right]\left[\bar{f}_{j}(H \bar{h})\right]
$$

which can give the down-type quark masses $\lambda_{i j}^{3} \frac{v_{1}<N_{H}><\bar{N}_{\bar{H}}>}{M_{*}^{2}} \bar{d}_{R j} d_{L i}$ and the neutrino Dirac masses $\lambda_{i j}^{4} \frac{v_{2}<N_{H}><\bar{N}_{\bar{H}}>}{M_{*}^{2}} N_{i} \nu_{j}$, respectively. However, the effects from the dimension-6 nonrenormalizable operators can be safely neglected if taking the cutoff scale of the theory high enough. For instance, taking $M_{*}=M_{P l}$ implies that their effects are suppressed by $M_{G U T}^{2} / M_{P l}^{2} \approx$ $10^{-4}$. So, the predictions described in Eq. (25) are not affected by these operators ${ }^{\dagger}$. Although there are also RGE effects on the mixing matrix, as observed for the normal hierarchical case, the RGE effects are mild [32]. In this paper, we will therefore use the mixing matrices established in the low energy phenomena above the see-saw scale.

We conclude for this subsection that the bilarge neutrino mixing can be well accomodated in the lepton Yukawa couplings in the flipped SU(5) model. The point is that for flipped $S U(5)$ model, the up-type quark mass matrix and the lepton mass matrix are arbitrary, and the symetric Majorana mass matrix for left-handed neutrino is arbitrary after the right-handed neutrinos decouple. Therefore, although the mass matrix for down-type quark is symmetric, we do have enough degrees of freedoms to produce the correct GUT scale SM fermion masses, CKM matrix and neutrino mixing matrix.

\subsection{One Loop Radiative Corrections of Sfermion Masses}

In order to establish notations and see where the difference of phenomenological consequences between the flipped and conventional SU(5) comes from, let us first illustrate a little on how the flavor changing terms arise in the supersymmetric theories. In this and next subsections, we shall use some useful conventions and definitions in the Minimal Supersymmetric Standard Model (MSSM) plus the right-handed neutrino fields $(\mathrm{MSSM}+\mathrm{N})$ given in Appendix A. In $\mathrm{MSSM}+\mathrm{N}$, the superpotential can be written as follows

$$
W_{S S M}=D_{i}^{c}\left(Y_{D}\right)_{i j} Q_{j} H_{1}+U_{i}^{c}\left(Y_{U}\right)_{i j} Q_{j} H_{2}+E_{i}^{c}\left(Y_{E}\right)_{i j} L_{j} H_{1}
$$

\footnotetext{
${ }^{\dagger}$ The dimension-5 operators can have larger effects. We have used one to generate the right-handed neutrino masses. More operators, like $\left(F_{i} \bar{f}_{\bar{h}}\right) F_{j} H / M_{*}$ and $\left(F_{i} \bar{H}\right)\left(\bar{f}_{j} f_{h}\right) / M_{*}$ can appear if we introduce one pair of Higgs $f_{h}=(5,3)$ and $\bar{f}_{\bar{h}}=(\overline{5},-3)$ under $S U(5) \times U(1)_{X}$. However, one may reintroduce the dimension-5 proton decay operators via the higgsino exchange. So, we are not going to consider this possibility.
} 


$$
+N_{i}\left(Y_{N}\right)_{i j} L_{j} H_{2}+\mu H_{1} H_{2}+\frac{1}{2}\left(M_{N}\right)_{i j} N_{i} N_{j} .
$$

$H_{2}$ and $H_{1}$ are the Higgs doublets which give the Dirac masses to the up-type quark (neutrino) and down-type quark (lepton), respectively. Assuming the universal SUSY breaking at high energy scale, we can get the radiative corrections to the mass of squark doublets, $m_{Q}^{2}$ (see Appendix A for the convention), with the following form [28]

$$
\delta m_{Q}^{2} \propto Y_{U}^{\dagger} Y_{U}+Y_{D}^{\dagger} Y_{D} .
$$

This just tells us that two couplings of $Q$ in the Eq. (29) both contribute. After the electroweak (EW) symmetry breaking, $m_{Q}^{2}$ gives $m_{U_{L}}^{2}$ and $m_{D_{L}}^{2}$. CKM mixing in the charged current interaction is obtained after diagonalising $Y_{U}$ and $Y_{D}$. Typically for the left-handed quark fields, the diagonalization is concerning $Y_{U}^{\dagger} Y_{U}$ and $Y_{D}^{\dagger} Y_{D}$. As can be seen clearly we will have misalignment between the quark and the squark mass matrix. Transforming for example the down-type squark fields simultaneously with the down-type quarks can diagonalise $Y_{D}^{\dagger} Y_{D}$ but can not diagonalise $m_{Q}^{2}$. Similarly for the up-type squarks. Thus, we have

$$
\begin{aligned}
\delta\left(\widetilde{m}_{U_{L}}^{2}\right)_{i j} & \propto\left(K y_{D}^{2} K^{\dagger}\right)_{i j} \approx K_{i 3} y_{b}^{2} K_{j 3}^{*} \\
\delta\left(\widetilde{m}_{D_{L}}^{2}\right)_{i j} & \propto\left(K^{\dagger} y_{U}^{2} K\right)_{i j} \approx K_{3 i}^{*} y_{t}^{2} K_{3 j} .
\end{aligned}
$$

where $y_{U}$ and $y_{D}$ are the diagonalised Yukawa couplings and $K$ is the CKM matrix. $\widetilde{m}^{2}$ is the mass squared written in the super-CKM base in which $Y_{U, D, E}$ are all diagonalised and the sfermion fields are transformed simultaneously with the fermion fields (see Appendix A). In the super-CKM base, we can see directly the extra flavor structures in the off-diagonal terms of these soft SUSY breaking masses. One of the main features of these corrections is that they are proportional to the corresponding flavor changing CKM matrix element. Typically $\delta\left(\widetilde{m}_{U_{L}}^{2}\right)_{i j}$ is given by the third column of the CKM matrix and $\delta\left(\widetilde{m}_{D_{L}}^{2}\right)_{i j}$ is given by the third row.

Similar story happens in the flipped $S U(5)$ model. As can be seen in Eqs. (11) and (13), $Y_{U}$ and $Y_{E}$ come seperately from the Yukawa couplings of five representation to the ten representation and of the five to the singlet representation. Since these two couplings both give the SM fermion Yukawa couplings, after transforming to the super-CKM base we can similarly get $\delta\left(\widetilde{m}_{U_{R}}^{2}\right)_{i j}$ and $\delta\left(\widetilde{m}_{E_{L}}^{2}\right)_{i j}$ radiatively corrected by the different elements of the same matrix. The story is different with the conventional $S U(5)$ embedding. In the conventional $S U(5)$ theory $10=\left(Q, U^{c}, E^{c}\right), \overline{5}=\left(D^{c}, L\right)$ and $1=N . Y_{D}$ and $Y_{E}$ both come from the Yukawa couplings of five representation to the ten representation. We have $\delta\left(\widetilde{m}_{D_{R}}^{2}\right)_{i j}$ and $\delta\left(\widetilde{m}_{E_{L}}^{2}\right)_{i j}$ being radiatively corrected by the coupling of five to the singlet representation which is basically the Yukawa coupling of the right-handed neutrino. Consequently $\delta\left(\widetilde{m}_{D_{R}}^{2}\right)_{i j}$ and $\delta\left(\widetilde{m}_{E_{L}}^{2}\right)_{i j}$ have similar dependences on the same matrix. Thus, what happens in the flipped $S U(5)$ is not quite similar to what happens in the conventional $S U(5)$ theory. And we have new phenomena in the charm and top quark physics, which will be discussed in detail in the next subsection and section III.

\subsection{Low Energy Implications}

We can define the SUSY breaking soft terms for the multiplets of the flipped $S U(5)$ model as follows

$$
-\Delta \mathcal{L}=\widetilde{F}_{i}^{*}\left(m_{10}^{2}\right)_{i j} \widetilde{F}_{j}+\widetilde{\widetilde{f}}_{i}^{*}\left(m_{\overline{5}}^{2}\right)_{i j} \widetilde{\bar{f}}_{j}+\tilde{l}_{i}^{* *}\left(m_{1}^{2}\right)_{i j} \tilde{l}_{j}^{c}+m_{h}^{2} \widetilde{h}^{*} \widetilde{h}+m_{\bar{h}}^{2} \widetilde{\bar{h}}^{*} \widetilde{\bar{h}}
$$




$$
\begin{aligned}
& +\left[\frac{1}{8} \widetilde{F}_{i}\left(Y_{10}^{A}\right)_{i j} \widetilde{F}_{j} \widetilde{h}_{i} \widetilde{F}_{i}\left(Y_{\overline{5}}^{A}\right)_{i j} \widetilde{\bar{f}}_{j} \widetilde{\bar{h}}_{i} \tilde{l}_{i}^{c}\left(Y_{1}^{A}\right)_{i} \widetilde{\bar{f}}_{j} \widetilde{h}^{\prime}+\mu B \widetilde{h} \widetilde{\bar{h}}\right. \\
& \left.+\frac{1}{2} M_{5} \lambda_{5} \lambda_{5}+\frac{1}{2} M_{X} \lambda_{X} \lambda_{X}+\text { h.c. }\right] .
\end{aligned}
$$

$\lambda_{5}$ and $\lambda_{X}$ are respectively the gaugino fields of the $S U(5)$ and $U(1)_{X}$ gauge groups. $M_{5}$ and $M_{X}$ are the gaugino masses. At $M_{G U T}$ scale, the matching conditions are

$$
\begin{gathered}
Y_{U}^{A}=\left(Y_{\overline{5}}^{A}\right)^{T}, \quad Y_{D}^{A}=Y_{10}^{A}, \quad Y_{N}^{A}=Y_{\overline{5}}^{A}, \quad Y_{E}^{A}=Y_{1}^{A} \\
m_{Q}^{2}=m_{10}^{2}, \quad m_{D}^{2}=\left(m_{10}^{2}\right)^{T}, \quad m_{U}^{2}=\left(m_{\overline{5}}^{2}\right)^{T}, \quad m_{L}^{2}=m_{\overline{5}}^{2}, \quad m_{E}^{2}=\left(m_{1}^{2}\right)^{T} .
\end{gathered}
$$

$\widetilde{h}$ et.al refer the scalar part of the corresponding superfields.

Similar to that in the MSSM, we do have $m_{5}^{2}$ corrected by the presence of two Yukawa couplings, $Y_{5}$ and $Y_{1}$ :

$$
\delta m_{\overline{5}}^{2} \propto c_{1} Y_{\overline{5}}^{\dagger} Y_{\overline{5}}+c_{2} Y_{1}^{\dagger} Y_{1}=c_{1}\left(Y_{U} Y_{U}^{\dagger}\right)^{T}+c_{2} Y_{E}^{\dagger} Y_{E}
$$

$c_{1,2}$ are coefficients. Again similar to what happend in the MSSM, $Y_{\overline{5}}$ and $Y_{1}$ give masses to the different SM fermions, i.e., the up-type quarks and leptons. The diagonalisations are concerning $Y_{U} Y_{U}^{\dagger}$ for the right-handed up-type quark and $Y_{E}^{\dagger} Y_{E}$ for the left-handed lepton fields. The resulting extra flavor structures in the sfermion mass matrices of right-handed uptype squarks and sleptons depend on the different elements of the same matrix. The results are detailed in the Appendix C. According to them, we can get the mass insertion parameters for the corresponding off-diagonal mass terms. Mass insertion parameter $\delta_{i j}$ is defined as $\delta_{i j}=M_{i j}^{2} / M_{\tilde{f}}^{2}$, where $M_{\tilde{f}}^{2}$ is the averaged mass squared of the $i$ and $j$ diagonal entries of the mass matrix of $\tilde{f}$. As an example, we have

$$
\begin{aligned}
& \left(\delta_{R R}^{U}\right)_{12} \approx-0.01 \frac{3 m_{0}^{2}+\left|A_{0}\right|^{2}}{\left|M_{\frac{1}{2}}\right|^{2}\left(1+0.16 m_{0}^{2} /\left|M_{\frac{1}{2}}\right|^{2}\right)}\left(U_{N}\right)_{13}^{*} y_{\tau}^{2}\left(U_{N}\right)_{23}, \\
& \left(\delta_{L L}^{E}\right)_{12} \approx-0.25 \frac{3 m_{0}^{2}+\left|A_{0}\right|^{2}}{0.6\left|M_{\frac{1}{2}}\right|^{2}+m_{0}^{2}}\left(U_{N}\right)_{31}^{*} y_{t}^{2}\left(U_{N}\right)_{32}\left(1+\frac{1}{40} \ln \frac{M_{G U T}^{2}}{M_{N}^{2}}\right) .
\end{aligned}
$$

In the expression, we have approximated $\left(\widetilde{m}_{f_{L L, R R}}^{2}\right)_{i i} \approx 6\left|M_{\frac{1}{2}}\right|^{2}+m_{0}^{2}(i=1,2$ and $f=U)$ and $\left(\widetilde{m}_{E_{L L, R R}}^{2}\right)_{j j} \approx 0.6\left|M_{\frac{1}{2}}\right|^{2}+m_{0}^{2}$. D-term contributions after the EW symmetry breaking have been neglected in the estimate. We have also taken $\ln \frac{M_{*}^{2}}{M_{G U T}^{2}}=10$ for the estimate which means that $M_{*} \doteq 3 \times 10^{18} \mathrm{GeV} \approx M_{P l}$ for $M_{G U T} \doteq 2.0 \times 10^{16} \mathrm{GeV}$. For $\delta_{L L}^{E}$ we have included the contributions from the RGE running between the $M_{G U T}$ scale and the $M_{N}$ scale shown in the Appendix B. We see that the corrections to $\delta_{L L}^{E}$ can be quite large if the relevant entries in $U_{N}$ can be of order one.

We can see clearly that typically $\delta_{L L}^{E}$ probes the third column of the matrix $U_{N}$, and $\delta_{L L}^{U}$ probes the third row of $U_{N}$. Similarly for $\delta_{L R}^{E}$ and $\delta_{L R}^{U}$. Notice that to have sizeable effects for the $\delta_{R R}^{U}$, we need large $\tan \beta$ because it is corrected by the presence of lepton Yukawa couplings. We can classify three typical scenarios by noticing that $U_{N}=V_{N_{L}}^{\dagger} V_{E_{L}}$ : 
(i) Neutrino mixings are all from $U_{N}$ and we can take $U=U_{N}^{\dagger}$ (see Eq. (A.7) for comparison).

(ii) Only the atmospheric neutrino mixing comes from $U_{N}$ and off-diagonal entries of the first row and first column of $U_{N}$ are all small and negligible;

(iii) No large off-diagonal entries in $U_{N}$ and large mixing angles come from $V_{N_{R}}$ and $M_{N}$.

The first possibility is supported by our discussions in the last subsection which can give the interesting phenomenological predictions. We will concentrate on the first case and comment on the second and the third one in the last section of the paper.

For scenario (i), we have

$$
\begin{aligned}
& \left(\delta_{R R}^{U}\right)_{i j} \propto U_{3 i} U_{3 j}^{*}, \quad\left(\delta_{L R}^{U}\right)_{3 k} \propto U_{33} U_{3 k}^{*}, \\
& \left(\delta_{L L}^{E}\right)_{i j} \propto U_{i 3} U_{j 3}^{*}, \quad\left(\delta_{L R}^{E}\right)_{k 3} \propto U_{k 3} U_{33}^{*}, \quad i \neq j, k \neq 3 .
\end{aligned}
$$

As expected, the corrections are concerning the right-handed squark and the left-handed sleptons. As we know, elements in the third row of the matrix $U$ are of the same order because of the bilarge mixing, then we have

$$
\left|\left(\delta_{R R}^{U}\right)_{12}\right| \sim\left|\left(\delta_{R R}^{U}\right)_{13}\right| \sim\left|\left(\delta_{R R}^{U}\right)_{23}\right| .
$$

In addition, in the case of large $\tan \beta$ for which $y_{\tau}$ and $y_{t}$ are of comparable magnitudes we have the following features for the moderate vaules of $M_{\frac{1}{2}}, m_{0}$ and $A_{0}$ :

(a) $\left(\delta_{R R}^{U}\right)_{i j}$ is of order $10^{-2}$ and $\left(\delta_{L L}^{E}\right)_{23}$ is of the order of $10^{-1}$;

(b) $\left(\delta_{L R}^{E}\right)_{23}$ is further suppressed by the VEV, $v_{1}=v \cos \beta$, and is of the order $10^{-2}-10^{-3}$ depending on the sfermion mass spectrum and is of the same order as $\left(\delta_{L R}^{U}\right)_{3 k}$;

(c) $\left(\delta_{L L}^{E}\right)_{13,12}$ and $\left(\delta_{L R}^{E}\right)_{13}$ are further suppressed by the presence of the small magnitude of $U_{13}\left(\right.$ or $\left.U_{e 3}\right)$.

\section{Phenomenology}

\subsection{Lepton Flavor Violation}

Satisfying the precision $\mu \rightarrow e \gamma$ constraint

$$
\operatorname{Br}(\mu \rightarrow e \gamma)<1.2 \times 10^{-11},
$$

puts order $10^{-3}$ upper bound on $\left(\delta_{L L}^{E}\right)_{12}$ and order $10^{-5}$ upper bound on $\left(\delta_{L R}^{E}\right)_{12}[33,34]$. For moderate values of $M_{\frac{1}{2}}, m_{0}$ and $A_{0}$, they correspond to having $\left|U_{e 3}\right| \lesssim 10^{-3}$. For the scenario discussed in the section 2.1 , they correspond to having $\epsilon \lesssim 10^{-3}$. We can simply satisfy this constraint by assuming $U_{e 3}=s_{13}=0$ in the matrix $U$. Similar thing happens to $\tau \rightarrow e+\cdots$ processes. 
Since the $\left(\delta_{L L}^{E}\right)_{23}$ is at the order of $10^{-1}$, we are able to get the promising prediction on $\tau \rightarrow \mu \gamma$ through the $\tan \beta$ enhanced contributions [16, 18]. A rough estimate in the mass insertion [18] shows that

$$
B r(\tau \rightarrow \mu \gamma) \approx 4 . \times 10^{-7} \times\left(\frac{1000 \mathrm{GeV}}{m_{\tilde{l}}}\right)^{4} \times\left(\frac{\tan \beta}{50}\right)^{2}
$$

for $m_{0}=\left|A_{0}\right|=2\left|M_{\frac{1}{2}}\right|$. The prediction is close to the present $1.1 \times 10^{-6}$ upper bound [37]. In the formulae, $m_{\tilde{l}}$ is the average mass of the $\tau$ and $\mu$ sleptons. And we have used $M_{N_{3}}=5.4 \times 10^{14}$ $\mathrm{GeV}$ to get $m_{\nu_{3}}=0.05 \mathrm{eV}$. If taking for example $\left|A_{0}\right|=6\left|M_{\frac{1}{2}}\right|$ and $m_{0}=2\left|M_{\frac{1}{2}}\right|$ (see Eq. (34)) which are in the region allowed by the constraints from the relic density of cold dark matter as well as $(g-2)_{\mu}$ and $b \rightarrow s \gamma[39]$, the $\left(\delta_{L L}^{E}\right)_{23}$ can be at the order of one. This will make $\operatorname{Br}(\tau \rightarrow \mu \gamma)$ beyond the experimental upper bound. However, as pointed out in ref. [40], there are some regions of parameter space where partial cancellation between contributions of Feynman diagrams happens. The cancellation can reduce $\operatorname{Br}(\tau \rightarrow \mu \gamma)$ significantly, e.g., by a factor of $10^{-1}-10^{-2}$, depending on sparticle mass spectrum. Therefore, in these regions $\left(\delta_{L L}^{E}\right)_{23}$ of order one and a low mass spectrum (say, below $1 \mathrm{TeV}$ ) are allowed by the upper bound of $\operatorname{Br}(\tau \rightarrow \mu \gamma)$

\subsection{B Physics}

B meson physics has been able to put good constraint on $\delta_{L L, R R, L R}^{D}$ to the order $10^{-2}[33$, 34]. These flavor changing structures contribute mainly through gluino loops. There are also processes, e.g., $b \rightarrow s \gamma, b \rightarrow d(s) l^{+} l^{-}, B \rightarrow l^{+} l^{-}$and $B_{d, s}-\bar{B}_{d, s}$ mixing for which chargino loops contribute a lot. Let's figure out which entries in $\delta^{U}$ are relevant to B physics. First of all, $\left(\delta_{R R}^{U}\right)_{i j}(i \neq j)$ are always irrelevant to the processes because the couplings involved with the right-handed up-type squarks are proportional to the Yukawa couplings and their contributions can be neglected because of the small up and charm Yukawa couplings. Due to the same reason for $\delta_{L R}^{U}$, the relevant entries are $\left(\delta_{L R}^{U}\right)_{13,23}$, which refer that the right-handed stop is propogating in the loop and give us large top Yukawa in the vertex.

Since flipped $S U(5)$ gives predictions on $\left(\delta_{L R}^{U}\right)_{31,32}$ and $\delta_{R R}^{U}$, not on $\left(\delta_{L R}^{U}\right)_{13,23}$ and $\delta_{L L}^{U}$, we do not have strong predictions on the B physics. This is a nice feature of the model. If flipped $S U(5)$ could give $10^{-2}$ prediction on $\left(\delta_{L R}^{U}\right)_{13}$, the double-penguin contributions to the $B_{d}-\bar{B}_{d}$ mixing would easily be as large as the SM prediction [35]. Without these extra flavor mixing entries, we simply go back to the conclusion made in [35]: the $\Delta M_{B_{s}}^{D P} / \Delta M_{B_{s}}^{S M}$ can be of order 1 while $\Delta M_{B_{d}}^{D P} / \Delta M_{B_{d}}^{S M} \sim \frac{1}{30} \Delta M_{B_{s}}^{D P} / \Delta M_{B_{s}}^{S M}$ and can satisfy the experimental bounds. Here $\Delta M^{D P}$ means the mass difference given by the double-penguin diagram and $\Delta M^{S M}$ is the mass difference given by the SM.

\section{3 $D-\bar{D}$ Mixing}

In the SM, the flavor changing transitions involving external up-type quarks, which are due to effective flavor changing neutral current (FCNC) couplings generated at loop level, are much more suppressed than those involving external down-type quarks. The effects for external uptype quarks are derived from the virtual exchanges of down-type quarks in a loop for which GIM mechanism [36] is much more effective because the mass splittings among down-type quarks 
are much less than those among up-type quarks. In the SM model, $D-\bar{D}$ mixing is extremely small and highly GIM suppressed by the factor $m_{s}^{2} / m_{W}^{2}\left(K_{u s} K_{c s}^{*}\right)^{2}$ (of order $10^{-8}$ ) which makes the SM prediction of order of $10^{6} \hbar s^{-1}$ (the contribution from the bottom loop is smaller because of the smaller CKM mixing).

However, the GIM mechanism is in general not valid in SUSY theories. In the scenarios of the minimal flavor violation for which flavor mixings are described by the CKM mixing, the SUSY contribution is suppressed by the degeneracy of the first and second generation squark masses and is of the same order of the SM contribution. However, with the misalignment of quark and squark mass matrices in a general SUSY theory, a sizeable $\left(\delta_{R R, L R}^{U}\right)_{12}$ can have significant effects on the $D-\bar{D}$ mixing through the gluino box diagram. The prediction on the mass difference, $\Delta M_{D}$, is proportional to $\left(\delta_{12}\right)^{2}$ for the $C P$ conserving case. The present upper bound [37] from the CLEO collaboration

$$
\Delta M_{D}<7 \times 10^{10} \hbar s^{-1}, 95 \% \mathrm{CL}
$$

has been able to put a $10^{-1}-10^{-2}$ constraint on $\left(\delta_{L L, R R, L R}^{U}\right)_{12}$ [38] depending on the squark and gluino mass scale.

In the framework of the constrained MSSM with for example gravity-mediated SUSY breaking at the high energy scale, we have indeed the radiatively generated misalignment in the quark and squark mass matrices as shown in Eq. (30). However, the relevant $\widetilde{m}_{12}^{2}$ is too small because it is corrected by the small entries of the CKM matrix (actually for this entry the contribution from the strange quark Yukawa coupling is larger but still too small to be interesting).

In the flipped $S U(5)$ model as we discussed in the subsection 2.2, bilarge neutrino mixing can give $10^{-2}$ right-handed up-type squark mixing and from which we can get

$$
\Delta M_{D} \approx 0.7 \times 10^{9} \times \frac{(1000 \mathrm{GeV})^{2}}{m_{\tilde{q}}^{2}} \times\left(\frac{\tan \beta}{50}\right)^{4} \hbar s^{-1},
$$

if assuming $m_{0}=\left|A_{0}\right|=2\left|M_{\frac{1}{2}}\right|$ which means $m_{\tilde{g}}^{2} / m_{\tilde{q}}^{2} \approx 0.6 . m_{\tilde{q}}^{2}$ in the unit $\mathrm{GeV}^{2}$ is the averaged right-handed up and charm squark mass squared, and $m_{\tilde{g}}$ is the gluino mass. We have used the data in Eq. (1) as inputs. Typically our prediction is one or two orders of magnitude smaller than the present bound which will be accessible at the CLEO-c and BES-III experiment.

As shown in the subsection 3.1, the prediction on $\operatorname{Br}(\tau \rightarrow \mu \gamma)$ is close to the present bound. Although $\Delta M_{D}$ has different dependences on the mass spectrum and $\tan \beta$ than $\operatorname{Br}(\tau \rightarrow \mu \gamma)$, we may expect that the present upper bound on $\Delta M_{D}$ is hard to be reached in quite a large part of the parameter space. In the Fig. 1, we show the correlation between the $\operatorname{Br}(\tau \rightarrow \mu \gamma)$ and $\Delta M_{D}$ in the model. We used the mass insertion approxiamted formular [16] to calculate the $\tan \beta$ enhanced $\operatorname{Br}(\tau \rightarrow \mu \gamma)$. We used $200 \mathrm{GeV}<m_{0},\left|M_{\frac{1}{2}}\right|,\left|A_{0}\right|<1.2 \mathrm{TeV}$ and $10<$ $\tan \beta<60$. One can see that $\Delta M_{D}$ can reach $10^{9} \hbar s^{-1}$ consistent with the constraint on $\operatorname{Br}(\tau \rightarrow \mu \gamma)$. There are also some points for which $\Delta M_{D}$ can reach $10^{10} \hbar s^{-1}$ and $\operatorname{Br}(\tau \rightarrow \mu \gamma)$ is smaller than the experimental bound by a factor of $10^{-1}-10^{-2}$, which corresponds to the case of cancellation discussed in subsection 3.1. In the plot, we have included the $2 \sigma$ constraints $0.0002<B r(b \rightarrow s \gamma)<0.00045, \Delta a_{\mu}^{S U S Y}<32 \times 10^{-10}$, and the $90 \% C L$ constraint $\operatorname{Br}\left(B_{s} \rightarrow \mu^{+} \mu^{-}\right)<2 . \times 10^{-6}$. Although these observables are also sensitive to large $\tan \beta$, they are actually not quite important for the model under study simply because the strong prediction on $\operatorname{Br}(\tau \rightarrow \mu \gamma)$ in quite a large part of the parameter space has constrained the mass spectrum to be around $1 \mathrm{TeV}$ and makes other constraints easily satisfied. 


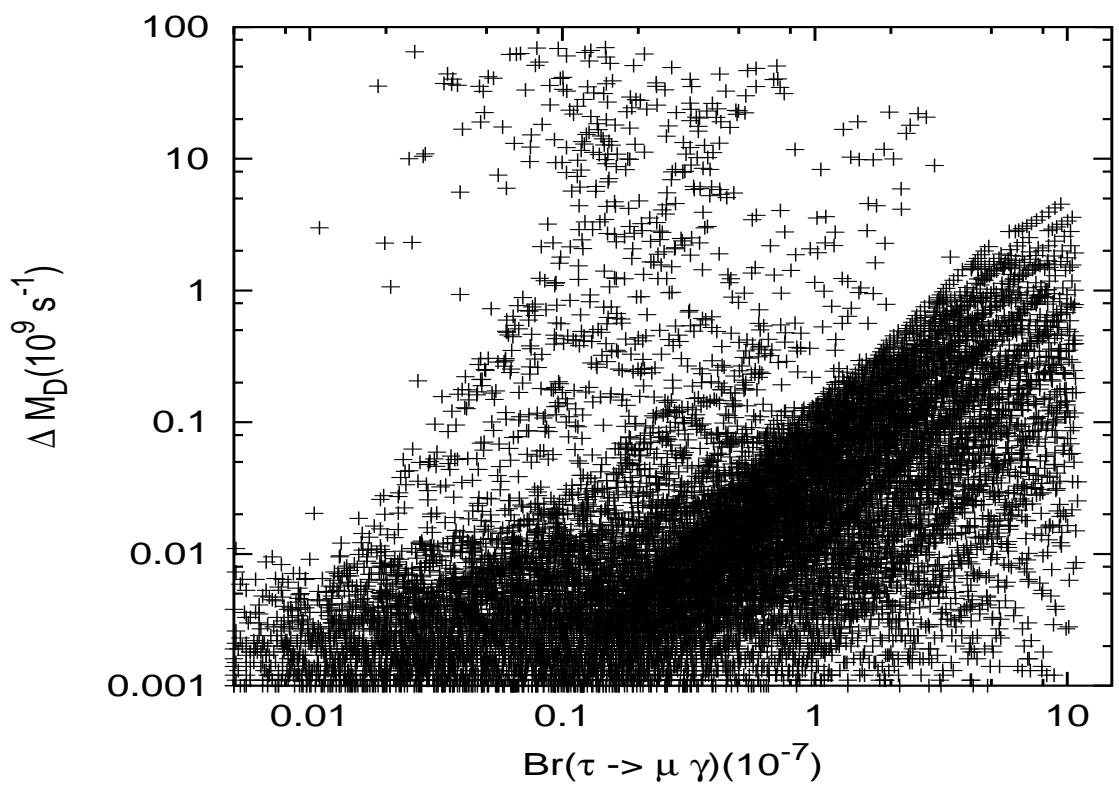

Figure 1: The correlation between the $B r(\tau \rightarrow \mu \gamma)$ and $\Delta M_{D}$ for the parameter space $200 \mathrm{GeV}<$ $m_{0},\left|M_{\frac{1}{2}}\right|,\left|A_{0}\right|<1.2 \mathrm{TeV}$, and $10<\tan \beta<60$. The constraint $\operatorname{Br}(\tau \rightarrow \mu \gamma)<1.1 \times 10^{-6}(90 \% C L)$ has been put.

\subsection{Top Physics}

In the SM, the tc transition rate is also very much GIM suppressed. The FCNC top quark decays, for example, $t \rightarrow c V \quad(V=\gamma, Z, g)$ and $t \rightarrow c h^{0}$, have branching ratios

$$
\begin{aligned}
\operatorname{Br}(t \rightarrow c \gamma) & \sim 5 \times 10^{-13} \\
\operatorname{Br}(t \rightarrow c Z) & \sim 1 \times 10^{-13} \\
\operatorname{Br}(t \rightarrow c g) & \sim 4 \times 10^{-13} \\
\operatorname{Br}\left(t \rightarrow c h^{0}\right) & \lesssim 10^{-13}\left(\text { depended on } \mathrm{m}_{\mathrm{h}^{0}}\right)
\end{aligned}
$$

which are unobservablly small [41, 42].

In SUSY theories, GIM mechanism is in general not valid and sizeable $\left(\delta_{R R, L R}^{U}\right)_{23}$ can have significant effects on the FCNC top to charm transition due to the gluino-mediated contributions (SUSY-QCD contributions). For $t \rightarrow c V \quad(V=\gamma, Z, g)$ and $t \rightarrow c h \quad\left(h=h^{0}, H^{0}, A^{0}\right)$ decays, as pointed out in Ref. [43], by changing $\delta_{23}^{U}$ by 3 orders of magnitude the branching ratios increase by 6 orders of magnitude due to the quadratic dependence of the branching ratios on the mixing coefficients. Typically for $\delta_{23}^{U} \sim 0.4$ and a light sparticle spectrum around $200 \mathrm{GeV}$, one can get

$$
\begin{aligned}
& B r(t \rightarrow c h) \simeq 10^{-4}, \\
& B r(t \rightarrow c g) \lesssim 10^{-5} .
\end{aligned}
$$

In the SM $t \rightarrow u$ processes are also GIM suppressed and have negligible predictions. The radiatively induced $t \rightarrow u, c$ couplings by the new physics beyond the SM can be probed by the 
top decay processes. Among them $t \rightarrow u, c+h^{0}$ can be probed by the top decay. $t \rightarrow u, c+g$ couplings can be probed by the single top production processes at the Tevatron or LHC:

$$
q \bar{q} \rightarrow \bar{u}, \bar{c}+t, \quad g g \rightarrow \bar{u}, \bar{c}+t .
$$

There are also direct top quark production processes

$$
u g \rightarrow t, \quad c g \rightarrow t
$$

For the tug coupling, LHC can reach the sensitivity equivalent to branching ratio $B r(t \rightarrow u g) \sim$ $10^{-6}$. And for the $t c g$ coupling, LHC can reach the sensitivity equivalent to branching ratio $B r(t \rightarrow c g) \sim 10^{-5}[44]$. For $t \rightarrow c, u+h^{0}$, LHC can reach order $10^{-5}$ branching ratio at $3 \sigma$ [44].

Our predictions on $\delta_{R R, L R}^{U}$ are typically of the order $10^{-2}$ for moderate soft SUSY breaking parameters which is not large enough to have significant effects and to be observed at the LHC. In particluar, according to Eq. (38) the present $D-\bar{D}$ mixing constraint makes $\left(\delta_{R R, L R}^{U}\right)_{32,31}$ also constrained in the model under study. However, in some regions of the parameter space, as pointed out in subsection 3.1, we can saturate the $D-\bar{D}$ mixing constraint and have observable FCNC effects for the top quark physics. In particular, the $t \rightarrow u, c+h^{0}$ processes are able to reach $10^{-5}-10^{-6}$ branching ratio which can be observed at LHC for $100 \mathrm{fb}^{-1}$ of integrated luminosity.

$\delta_{R R, L R}^{U}$ can give rise to new contributions to more processes in top physics. For instance, they will have sizeable effects on $t \rightarrow c, u+l^{+} l^{-}$processes. These top quark FCNC couplings can also be probed in top and charm associated productions at linear and $\gamma-\gamma$ colliders [45].

\section{Conclusions and Discussions}

In summary, we have constructed a specific supersymmetric flipped SU(5) unification model in which bilarge neutrino mixing is incorporated in the lepton Yukawa couplings. Non-renormalizable operators have been introduced in the superpotential at the GUT scale in order to generate the right-handed neutrino Majorana masses. The effects of other non-renormalizable terms on the low energy implications can be safely neglected by taking the theoretical cutoff scale to be the Planck scale. The universal supersymmetry breaking is assumed at the Planck scale. Because the up-type and down-type quarks in the model are flipped in the representations ten and five with respect to the usual $\mathrm{SU}(5)$, the radiatively generated flavor mixing in the squark mass matrix due to the large neutrino mixing has a pattern different from those in the conventional $\mathrm{SU}(5)$ or $\mathrm{SO}(10)$ supersymmetric GUT. This leads to the phenomenological consequences quite different from $\mathrm{SU}(5)$ or $\mathrm{SO}(10)$ supersymmtric GUT. The left-handed slepton mixing is induced by the up-type quark Yukawa coupling which is not suppressed when $\tan \beta$ is small. The experimental bound on $\mu \rightarrow e \gamma$ can be safely satisfied by taking $\theta_{13}$ small enough. When $\tan \beta$ is large the branching ratio of the $\tau \rightarrow \mu \gamma$ is predicted to reach the present experimental bound with a mass spectrum around $1 \mathrm{TeV}$. Sizeable radiative corrections happen also to the righthanded up-type squark mass matrix if $\tan \beta$ is large $(\gtrsim 30)$. This radiatively generated flavor mixing has almost no impact on B physics. On the contrary, it has effects in top and charm physics. Because of the special feature of the flipped $S U(5)$ model, the flavor mixing involving the first generation up-type squark is never suppressed by small $\theta_{13}$. In the conventional $S U(5)$ model satisfying the $\mu \rightarrow e \gamma$ constraint makes the SUSY prediction on the $K-\bar{K}$ mixing 
negligible. However, similar thing never happens in the flipped $S U(5)$ model. In particular, we have shown that the radiatively generated falvor mixing can give a promising prediction on the $D-\bar{D}$ mixing. That is, for moderate values of the soft SUSY breaking parameters, $\Delta M_{D}$ is of one order of magnitude smaller than the present experimental upper bound. Because $\operatorname{Br}(\tau \rightarrow \mu \gamma)$ and $\Delta M_{D}$ have different dependences on the mass spectrum and $\tan \beta$ they are basically complementary observables to test the supersymmetric theory. For the predicted squark flavor mixing, $t \rightarrow u, c+h^{0}$ can reach $10^{-6}-10^{-7}$ branching ratio. In some regions of the parameter space $\Delta M_{D}$ can saturate the present bound. For these ranges of parameter space, $t \rightarrow u, c+h^{0}$ can reach $10^{-5}-10^{-6}$ which would be observed at the LHC and future $\gamma-\gamma$ colliders. However a light spectrum is basically required by having sizeable effects on the top quark FCNC processes. So the top quark FCNC processes are probably hard to be found at LHC for the scenario (i) that we have concentrated on in the paper. A detailed analysis including all the relevant experimental constraints is needed to make a definite conclusion on the discovery potential of rare top decays at LHC. Moreover the model has effects in FCNC D meson decays such as $D \rightarrow \rho(\pi) \gamma$, which is worth to be examined.

For the scenario (ii) in which only the atmospheric neutrino mixing is accommodated in the Yukawa coupling, we only have sizeable predictions on the $\left(\delta_{R R}^{U, E}\right)_{23,32},\left(\delta_{L R}^{U}\right)_{32}$ and $\left(\delta_{L R}^{E}\right)_{23}$. In principle $\left(\delta_{R R, L R}^{U}\right)_{32}$ can be quite large (even order one) if we have very large $A_{0}$. However, as happened for the scenario (i) the $\tau \rightarrow \mu+\gamma$ would limit them to be smaller than order of $10^{-1}$ in quite a large part of parameter space and consequently make the rare top FCNC decay hard to be observed at the LHC. We need detailed calculations to say definitely on the discovery potential.

The scenario (iii) is not interesting for $D-\bar{D}$ mixing and Top quark FCNC. However it may still be interesting for the physics of the lepton flavor violation[18]. The point is that by involving a low mass spectrum the $1 / m_{\tilde{l}}^{4}$ dependence of the branching ratio, as shown in Eq. (40), can compensate part of the suppression given by a small off-diagonal entry in $U_{N}$. Typically with an order of $10^{-2}$ off-diagonal entry and a low mass spectrum as for example $m_{\tilde{l}} \sim 200 \mathrm{GeV}$, $\operatorname{Br}(\tau \rightarrow \mu \gamma)$ can still reach $10^{-8}$ and be accessible in the near future experiments.

In the paper, we did not consider the possible Majorana phases in the discussions. These phases, which have no effects in the neutrino oscillation experiments, can indeed give effects on the radiative corrections to the squark and slepton mass matrices and lead to $C P$ violating effects in the low energy phenomena, e.g., the $C P$ violating effects in the lepton physics, $D-\bar{D}$ mixing and the top quark decay processes. It is possible that these phases can contribute to the electric dipole moment of the electron, muon and neutron [46] and are of much interests.

The flipped $S U(5)$ model can be embedded in the $S O(10)$ model and we would have similar implications on the flavor physics if $S O(10)$ takes the breaking chain through the flipped $S U(5)$. We leave this for the future work.

\section{Acknowledgements}

L.W would like to thank Vempati Sudhir and Oscar Vives for helpful communications on the lepton flavor violation. The research of C.-S. Huang was supported in part by the Natural Science Foundation of China. And the research of T. Li was supported by the National Science Foundation under Grant No. PHY-0070928. 


\section{Appendix A: Some Conventions in MSSM+N}

The soft SUSY breaking terms are written as

$$
\begin{aligned}
-\Delta \mathcal{L}= & \tilde{U}_{i}^{*}\left(m_{U}^{2}\right)_{i j} \tilde{U}_{j}+\tilde{D}_{i}^{*}\left(m_{D}^{2}\right)_{i j} \tilde{D}_{j}+\tilde{Q}_{i}^{*}\left(m_{Q}^{2}\right)_{i j} \tilde{Q}_{j}+\tilde{E}_{i}^{*}\left(m_{E}^{2}\right)_{i j} \tilde{E}_{j}+\tilde{N}_{i}^{*}\left(m_{N}^{2}\right)_{i j} \tilde{N}_{j} \\
& +\tilde{L}_{i}^{*}\left(m_{L}^{2}\right)_{i j} \tilde{L}_{j}+m_{H_{1}}^{2} \tilde{H}_{1}^{\dagger} \tilde{H}_{1}+m_{H_{2}}^{2} \tilde{H}_{2}^{\dagger} \tilde{H}_{2}+\left[\tilde{U}_{i}^{*}\left(Y_{U}^{A}\right)_{i j} \tilde{Q}_{j} \tilde{H}_{2}\right. \\
& \left.+\tilde{D}_{i}^{*}\left(Y_{D}^{A}\right)_{i j} \tilde{Q}_{j} \tilde{H}_{1}+\tilde{E}_{i}^{*}\left(Y_{E}^{A}\right)_{i j} \tilde{L}_{j} \tilde{H}_{1}+\tilde{N}_{i}\left(Y_{N}^{A}\right)_{i j} \tilde{L}_{j} \tilde{H}_{2}+B \mu \tilde{H}_{1} \tilde{H}_{2}+\text { h.c. }\right],
\end{aligned}
$$

and the gaugino masses are

$$
-\Delta \mathcal{L}=\frac{1}{2}\left(M_{1} \lambda_{1} \lambda_{1}+M_{2} \lambda_{2} \lambda_{2}+M_{3} \lambda_{3} \lambda_{3}\right)+\text { h.c. }
$$

where $\tilde{Q}$ et.al refer to the scalar parts of the corresponding superfields. $\lambda_{i}(i=1,2,3)$ are the gaugino fields for $U(1)_{Y}, S U(2)_{W}$ and $S U(3)_{C}$ groups. In the Appendices B and $\mathrm{C}$, we list the one-loop RGEs in SUSY model with right-handed neutrinos.

We can parametrize the Yukawa couplings in Eq.(29) as follows

$$
Y_{U}=V_{U_{R}} y_{U} V_{U_{L}}^{\dagger}, Y_{D}=V_{D_{R}} y_{D} V_{D_{L}}^{\dagger}, Y_{E}=V_{E_{R}} y_{E} V_{E_{L}}^{\dagger}, Y_{N}=V_{N_{R}} y_{N} V_{N_{L}}^{\dagger} .
$$

$V_{U_{R}}$ et.al are unitary matrices and $y_{U}$ et.al are diagonal and real matrices. After the electroweak symmetry breaking due to the vacuum expectation values of the $H_{1}$ and $H_{2}$ fields

$$
<H_{1}>=\left(\begin{array}{c}
0 \\
v_{1}
\end{array}\right), \quad<H_{2}>=\left(\begin{array}{c}
v_{2} \\
0
\end{array}\right)
$$

we redefine the fermion fields to diagonalise the $Y_{U}, Y_{D}$ and $Y_{E}$, and get the Dirac masses of the SM fermions. The CKM matrix is then obtained in the charged current interactions:

$$
K=V_{U_{L}}^{\dagger} V_{D_{L}}
$$

and we define

$$
U_{N}=V_{N_{L}}^{\dagger} V_{E_{L}}
$$

The left-handed neutrino mass matrix in the interaction eigenstate is

$$
\begin{aligned}
-\Delta \mathcal{L} & =\frac{1}{2} \overline{\nu^{c}} M_{\nu} \nu+h . c . \\
M_{\nu} & =v^{2} \sin ^{2} \beta V_{E_{L}}^{T} Y_{N}^{T} M_{N}^{-1} Y_{N} V_{E_{L}}=U^{*} m_{\nu} U^{\dagger},
\end{aligned}
$$

where $v^{2}=v_{1}^{2}+v_{2}^{2}$ and $\tan \beta=v_{2} / v_{1}$. Matrix $U$ is the one responsible for the neutrino mixing.

To see the extra flavor mixing in the squark mass matrix, we redefine the sfermion fields in the same way as the corresponding fermion fields and be in the so-called super-CKM base:

$$
\begin{gathered}
\left(\tilde{U}_{L}, u_{L}\right) \rightarrow V_{U_{L}}\left(\tilde{U}_{L}, u_{L}\right), \quad\left(\tilde{U}_{R}, u_{R}\right) \rightarrow V_{U_{R}}\left(\tilde{U}_{R}, u_{R}\right), \\
\left(\tilde{D}_{L}, d_{L}\right) \rightarrow V_{D_{L}}\left(\tilde{D}_{L}, d_{L}\right), \quad\left(\tilde{D}_{R}, d_{R}\right) \rightarrow V_{D_{R}}\left(\tilde{D}_{R}, d_{R}\right), \\
\left(\tilde{E}_{L}, e_{L}\right) \rightarrow V_{E_{L}}\left(\tilde{E}_{L}, e_{L}\right), \quad\left(\tilde{E}_{R}, e_{R}\right) \rightarrow V_{E_{R}}\left(\tilde{E}_{R}, e_{R}\right), \quad\left(\tilde{\nu}_{L}, \nu_{L}\right) \rightarrow V_{E_{L}}\left(\tilde{\nu}_{L}, \nu_{L}\right) .
\end{gathered}
$$


Squark and slepton mass squared matrices will be denoted as $\widetilde{m}^{2}$ in the super-CKM base. Trilinear terms will be denoted as $y^{A}$ (see Eq. (B.17) for definitions). The six by six squark mass matrices relevant for the low energy phenomenology are

$$
\widetilde{M}_{f}^{2}=\left(\begin{array}{cc}
\widetilde{M}_{f_{L L}}^{2} & \widetilde{M}_{f_{L R}}^{2} \\
\widetilde{M}_{f_{L R}}^{2 \dagger} & \widetilde{M}_{f_{R R}}^{2}
\end{array}\right), \quad f=U, D, E .
$$

Definitions of the relevant extries can be found in the Appendix B in which there are additional contributions from the $S U(2)_{L} \times U(1)_{Y}$ D-terms after the electroweak symmetry breaking.

\section{Appendix B: RGEs in the MSSM+N}

RGEs are derived as follows

$$
\begin{aligned}
2 \frac{d \tilde{Y}_{U}}{d t}= & \left(\frac{16}{3} \tilde{\alpha}_{3}+3 \tilde{\alpha}_{2}+\frac{13}{9} \tilde{\alpha}_{1}\right) \tilde{Y}_{U}-3\left(\tilde{Y}_{U} \tilde{Y}_{U}^{\dagger}+\operatorname{tr}\left(\tilde{Y}_{U} \tilde{Y}_{U}^{\dagger}\right)\right) \tilde{Y}_{U} \\
& -\tilde{Y}_{U} \tilde{Y}_{D}^{\dagger} \tilde{Y}_{D}-\operatorname{tr}\left(\tilde{Y}_{N} \tilde{Y}_{N}^{\dagger}\right) \tilde{Y}_{U} \\
2 \frac{d \tilde{Y}_{D}}{d t}= & \left(\frac{16}{3} \tilde{\alpha}_{3}+3 \tilde{\alpha}_{2}+\frac{7}{9} \tilde{\alpha}_{1}\right) \tilde{Y}_{D}-3\left(\tilde{Y}_{D}^{\dagger} \tilde{Y}_{D}+\operatorname{tr}\left(\tilde{Y}_{D} \tilde{Y}_{D}^{\dagger}\right)\right) \tilde{Y}_{D} \\
& -\tilde{Y}_{D} \tilde{Y}_{U}^{\dagger} \tilde{Y}_{U}-\operatorname{tr}\left(\tilde{Y}_{E} \tilde{Y}_{E}^{\dagger}\right) \tilde{Y}_{D} \\
2 \frac{d \tilde{Y}_{E}}{d t}= & \left(3 \tilde{\alpha}_{2}+3 \tilde{\alpha}_{1}\right) \tilde{Y}_{E}-3 \tilde{Y}_{E} \tilde{Y}_{E}^{\dagger} \tilde{Y}_{E}-\operatorname{tr}\left(\tilde{Y}_{E} \tilde{Y}_{E}^{\dagger}\right) \tilde{Y}_{E}-\tilde{Y}_{E} \tilde{Y}_{N}^{\dagger} \tilde{Y}_{N}-3 \operatorname{tr}\left(\tilde{Y}_{D} \tilde{Y}_{D}^{\dagger}\right) \tilde{Y}_{E}, \\
2 \frac{d \tilde{Y}_{N}}{d t}= & \left(3 \tilde{\alpha}_{2}+\tilde{\alpha}_{1}\right) \tilde{Y}_{N}-3 \tilde{Y}_{N} \tilde{Y}_{N}^{\dagger} \tilde{Y}_{N}-\operatorname{tr}\left(\tilde{Y}_{N} \tilde{Y}_{N}^{\dagger}\right) \tilde{Y}_{N}-\tilde{Y}_{N} \tilde{Y}_{E}^{\dagger} \tilde{Y}_{E}-3 \operatorname{tr}\left(\tilde{Y}_{U} \tilde{Y}_{U}^{\dagger}\right) \tilde{Y}_{N} .
\end{aligned}
$$

In the above formulae, we have $\tilde{\alpha}=\alpha / 4 \pi, \tilde{Y}=Y / 4 \pi, t=\ln \left(M_{G U T}^{2} / Q^{2}\right)$. For the SUSY breaking soft terms, we have

$$
\begin{aligned}
2 \frac{d \tilde{Y}_{U}^{A}}{d t}= & \left(\frac{16}{3} \tilde{\alpha}_{3}+3 \tilde{\alpha}_{2}+\frac{13}{9} \tilde{\alpha}_{1}\right) \tilde{Y}_{U}^{A}+2\left(\frac{16}{3} \tilde{\alpha}_{3} M_{3}+3 \tilde{\alpha}_{2} M_{2}+\frac{13}{9} \tilde{\alpha}_{1} M_{1}\right) \tilde{Y}_{U} \\
& -4 \tilde{Y}_{U} \tilde{Y}_{U}^{\dagger} \tilde{Y}_{U}^{A}-6 \operatorname{tr}\left(\tilde{Y}_{U}^{A} \tilde{Y}_{U}^{\dagger}\right) \tilde{Y}_{U}-5 \tilde{Y}_{U}^{A} \tilde{Y}_{U}^{\dagger} \tilde{Y}_{U}-3 \operatorname{tr}\left(\tilde{Y}_{U} \tilde{Y}_{U}^{\dagger}\right) \tilde{Y}_{U}^{A} \\
& -2 \tilde{Y}_{U} \tilde{Y}_{D}^{\dagger} \tilde{Y}_{D}^{A}-\tilde{Y}_{U}^{A} \tilde{Y}_{D}^{\dagger} \tilde{Y}_{D}-2 \operatorname{tr}\left(\tilde{Y}_{N}^{A} \tilde{Y}_{N}^{\dagger}\right) \tilde{Y}_{U}-\operatorname{tr}\left(\tilde{Y}_{N} \tilde{Y}_{N}^{\dagger}\right) \tilde{Y}_{U}^{A} \\
2 \frac{d \tilde{Y}_{D}^{A}}{d t}= & \left(\frac{16}{3} \tilde{\alpha}_{3}+3 \tilde{\alpha}_{2}+\frac{7}{9} \tilde{\alpha}_{1}\right) \tilde{Y}_{D}^{A}+2\left(\frac{16}{3} \tilde{\alpha}_{3} M_{3}+3 \tilde{\alpha}_{2} M_{2}+\frac{7}{9} \tilde{\alpha}_{1} M_{1}\right) \tilde{Y}_{D} \\
& -4 \tilde{Y}_{D} \tilde{Y}_{D}^{\dagger} \tilde{Y}_{D}^{A}-6 \operatorname{tr}\left(\tilde{Y}_{D}^{A} \tilde{Y}_{D}^{\dagger}\right) \tilde{Y}_{D}-5 \tilde{Y}_{D}^{A} \tilde{Y}_{D}^{\dagger} \tilde{Y}_{D}-3 \operatorname{tr}\left(\tilde{Y}_{D} \tilde{Y}_{D}^{\dagger}\right) \tilde{Y}_{D}^{A} \\
& -2 \tilde{Y}_{D} \tilde{Y}_{U}^{\dagger} \tilde{Y}_{U}^{A}-\tilde{Y}_{D}^{A} \tilde{Y}_{U}^{\dagger} \tilde{Y}_{U}-2 \operatorname{tr}\left(\tilde{Y}_{E}^{A} \tilde{Y}_{E}^{\dagger}\right) \tilde{Y}_{D}-\operatorname{tr}\left(\tilde{Y}_{E} \tilde{Y}_{E}^{\dagger}\right) \tilde{Y}_{D}^{A} \\
2 \frac{d \tilde{Y}_{E}^{A}}{d t}= & \left(3 \tilde{\alpha}_{2}+3 \tilde{\alpha}_{1}\right) \tilde{Y}_{E}^{A}+2\left(3 \tilde{\alpha}_{2} M_{2}+3 \tilde{\alpha}_{1} M_{1}\right) \tilde{Y}_{E} \\
& -4 \tilde{Y}_{E} \tilde{Y}_{E}^{\dagger} \tilde{Y}_{E}^{A}-2 \operatorname{tr}\left(\tilde{Y}_{E}^{A} \tilde{Y}_{E}^{\dagger}\right) \tilde{Y}_{E}-5 \tilde{Y}_{E}^{A} \tilde{Y}_{E}^{\dagger} \tilde{Y}_{E}-\operatorname{tr}\left(\tilde{Y}_{E} \tilde{Y}_{E}^{\dagger}\right) \tilde{Y}_{E}^{A}
\end{aligned}
$$




$$
\begin{aligned}
& -2 \tilde{Y}_{E} \tilde{Y}_{N}^{\dagger} \tilde{Y}_{N}^{A}-\tilde{Y}_{E}^{A} \tilde{Y}_{N}^{\dagger} \tilde{Y}_{N}-6 \operatorname{tr}\left(\tilde{Y}_{D}^{A} \tilde{Y}_{D}^{\dagger}\right) \tilde{Y}_{E}-3 \operatorname{tr}\left(\tilde{Y}_{D} \tilde{Y}_{D}^{\dagger}\right) \tilde{Y}_{E}^{A}, \\
& 2 \frac{d \tilde{Y}_{N}^{A}}{d t}=\left(3 \tilde{\alpha}_{2}+\tilde{\alpha}_{1}\right) \tilde{Y}_{N}^{A}+2\left(3 \tilde{\alpha}_{2} M_{2}+\tilde{\alpha}_{1} M_{1}\right) \tilde{Y}_{N} \\
& -4 \tilde{Y}_{N} \tilde{Y}_{N}^{\dagger} \tilde{Y}_{N}^{A}-2 \operatorname{tr}\left(\tilde{Y}_{N}^{A} \tilde{Y}_{N}^{\dagger}\right) \tilde{Y}_{N}-5 \tilde{Y}_{N}^{A} \tilde{Y}_{N}^{\dagger} \tilde{Y}_{N}-\operatorname{tr}\left(\tilde{Y}_{N} \tilde{Y}_{N}^{\dagger}\right) \tilde{Y}_{N}^{A} \\
& -2 \tilde{Y}_{N} \tilde{Y}_{E}^{\dagger} \tilde{Y}_{E}^{A}-\tilde{Y}_{N}^{A} \tilde{Y}_{E}^{\dagger} \tilde{Y}_{E}-6 \operatorname{tr}\left(\tilde{Y}_{U}^{A} \tilde{Y}_{U}^{\dagger}\right) \tilde{Y}_{N}-3 \operatorname{tr}\left(\tilde{Y}_{U} \tilde{Y}_{U}^{\dagger}\right) \tilde{Y}_{N}^{A}, \\
& \frac{d m_{U}^{2}}{d t}=\left(\frac{16}{3} \tilde{\alpha}_{3}\left|M_{3}\right|^{2}+\frac{16}{9} \tilde{\alpha}_{1}\left|M_{1}\right|^{2}\right)-\left(\tilde{Y}_{U} \tilde{Y}_{U}^{\dagger} m_{U}^{2}+m_{U}^{2} \tilde{Y}_{U} \tilde{Y}_{U}^{\dagger}\right) \\
& -2\left(\tilde{Y}_{U} m_{Q}^{2} \tilde{Y}_{U}^{\dagger}+\tilde{Y}_{U} \tilde{Y}_{U}^{\dagger} m_{H_{2}}^{2}+\tilde{Y}_{U}^{A} \tilde{Y}_{U}^{A \dagger}\right) \\
& \frac{d m_{D}^{2}}{d t}=\left(\frac{16}{3} \tilde{\alpha}_{3}\left|M_{3}\right|^{2}+\frac{4}{9} \tilde{\alpha}_{1}\left|M_{1}\right|^{2}\right)-\left(\tilde{Y}_{D} \tilde{Y}_{D}^{\dagger} m_{D}^{2}+m_{D}^{2} \tilde{Y}_{D} \tilde{Y}_{D}^{\dagger}\right) \\
& -2\left(\tilde{Y}_{D} m_{Q}^{2} \tilde{Y}_{D}^{\dagger}+\tilde{Y}_{D} \tilde{Y}_{D}^{\dagger} m_{H_{1}}^{2}+\tilde{Y}_{D}^{A} \tilde{Y}_{D}^{A \dagger}\right), \\
& \frac{d m_{Q}^{2}}{d t}=\left(\frac{16}{3} \tilde{\alpha}_{3}\left|M_{3}\right|^{2}+3 \tilde{\alpha}_{2}\left|M_{2}\right|^{2}+\frac{1}{9} \tilde{\alpha}_{1}\left|M_{1}\right|^{2}\right) \\
& -\frac{1}{2}\left(\tilde{Y}_{U}^{\dagger} \tilde{Y}_{U} m_{Q}^{2}+m_{Q}^{2} \tilde{Y}_{U}^{\dagger} \tilde{Y}_{U}\right)-\left(\tilde{Y}_{U}^{\dagger} m_{U}^{2} \tilde{Y}_{U}+\tilde{Y}_{U}^{\dagger} \tilde{Y}_{U} m_{H_{2}}^{2}+\tilde{Y}_{U}^{A \dagger} \tilde{Y}_{U}^{A}\right) \\
& -\frac{1}{2}\left(\tilde{Y}_{D}^{\dagger} \tilde{Y}_{D} m_{Q}^{2}+m_{Q}^{2} \tilde{Y}_{D}^{\dagger} \tilde{Y}_{D}\right)-\left(\tilde{Y}_{D}^{\dagger} m_{D}^{2} \tilde{Y}_{D}+\tilde{Y}_{D}^{\dagger} \tilde{Y}_{D} m_{H_{1}}^{2}+\tilde{Y}_{D}^{A \dagger} \tilde{Y}_{D}^{A}\right) \\
& \frac{d m_{N}^{2}}{d t}=-\left[m_{N}^{2}\left(\tilde{Y}_{N} \tilde{Y}_{N}^{\dagger}\right)^{T}+\left(\tilde{Y}_{N} \tilde{Y}_{N}^{\dagger}\right)^{T} m_{N}^{2}\right]-2\left(\tilde{Y}_{N} m_{L}^{2} \tilde{Y}_{N}^{\dagger}+\tilde{Y}_{N} \tilde{Y}_{N}^{\dagger} m_{H_{2}}^{2}+\tilde{Y}_{N}^{A} \tilde{Y}_{N}^{A \dagger}\right)^{T} \\
& \frac{d m_{E}^{2}}{d t}=4 \tilde{\alpha}_{1}\left|M_{1}\right|^{2}-\left(m_{E}^{2} \tilde{Y}_{E} \tilde{Y}_{E}^{\dagger}+\tilde{Y}_{E} \tilde{Y}_{E}^{\dagger} m_{E}^{2}\right)-2\left(\tilde{Y}_{E} m_{L}^{2} \tilde{Y}_{E}^{\dagger}+\tilde{Y}_{E} \tilde{Y}_{E}^{\dagger} m_{H_{1}}^{2}+\tilde{Y}_{E}^{A} \tilde{Y}_{E}^{A \dagger}\right) \\
& \frac{d m_{L}^{2}}{d t}=\left(3 \tilde{\alpha}_{2}\left|M_{2}\right|^{2}+\tilde{\alpha}_{1}\left|M_{1}\right|^{2}\right) \\
& -\frac{1}{2}\left(\tilde{Y}_{N}^{\dagger} \tilde{Y}_{N} m_{L}^{2}+m_{L}^{2} \tilde{Y}_{N}^{\dagger} \tilde{Y}_{N}\right)-\left(\tilde{Y}_{N}^{\dagger}\left(m_{N}^{2}\right)^{T} \tilde{Y}_{N}+\tilde{Y}_{N}^{\dagger} \tilde{Y}_{N} m_{H_{2}}^{2}+\tilde{Y}_{U}^{A \dagger} \tilde{Y}_{U}^{A}\right) \\
& -\frac{1}{2}\left(\tilde{Y}_{E}^{\dagger} \tilde{Y}_{E} m_{L}^{2}+m_{L}^{2} \tilde{Y}_{E}^{\dagger} \tilde{Y}_{E}\right)-\left(\tilde{Y}_{E}^{\dagger} m_{E}^{2} \tilde{Y}_{E}+\tilde{Y}_{E}^{\dagger} \tilde{Y}_{E} m_{H_{1}}^{2}+\tilde{Y}_{E}^{A \dagger} \tilde{Y}_{E}^{A}\right), \\
& \frac{d m_{H_{2}}^{2}}{d t}=\left(3 \tilde{\alpha}_{2}\left|M_{2}\right|^{2}+\tilde{\alpha}_{1}\left|M_{1}\right|^{2}\right) \\
& -3 \operatorname{tr}\left(\tilde{Y}_{U}^{\dagger} \tilde{Y}_{U} m_{H_{2}}^{2}+\tilde{Y}_{U}^{\dagger} m_{U}^{2} \tilde{Y}_{U}+\tilde{Y}_{U} m_{Q}^{2} \tilde{Y}_{U}^{\dagger}+\tilde{Y}_{U}^{A} \tilde{Y}_{U}^{A \dagger}\right) \\
& -\operatorname{tr}\left(\tilde{Y}_{N}^{\dagger} \tilde{Y}_{N} m_{H_{2}}^{2}+\tilde{Y}_{N}^{\dagger}\left(m_{N}^{2}\right)^{T} \tilde{Y}_{N}+\tilde{Y}_{N} m_{L}^{2} \tilde{Y}_{N}^{\dagger}+\tilde{Y}_{N}^{A} \tilde{Y}_{N}^{A \dagger}\right),
\end{aligned}
$$




$$
\begin{aligned}
\frac{d m_{H_{1}}^{2}}{d t}= & \left(3 \tilde{\alpha}_{2}\left|M_{2}\right|^{2}+\tilde{\alpha}_{1}\left|M_{1}\right|^{2}\right) \\
& -3 \operatorname{tr}\left(\tilde{Y}_{D}^{\dagger} \tilde{Y}_{D} m_{H_{1}}^{2}+\tilde{Y}_{D}^{\dagger} m_{D}^{2} \tilde{Y}_{D}+\tilde{Y}_{D} m_{Q}^{2} \tilde{Y}_{D}^{\dagger}+\tilde{Y}_{D}^{A} \tilde{Y}_{D}^{A \dagger}\right) \\
& -\operatorname{tr}\left(\tilde{Y}_{E}^{\dagger} \tilde{Y}_{E} m_{H_{1}}^{2}+\tilde{Y}_{E}^{\dagger} m_{E}^{2} \tilde{Y}_{E}+\tilde{Y}_{E} m_{L}^{2} \tilde{Y}_{E}^{\dagger}+\tilde{Y}_{E}^{A} \tilde{Y}_{E}^{A \dagger}\right) .
\end{aligned}
$$

In the super-CKM base, the Yukawa couplings of the SM fermions are all diagonalised and generate the SM fermions in the mass eigenstates. The SUSY breaking soft terms in this base can be written as

$$
\begin{aligned}
& \widetilde{m}_{U_{L}}^{2}=V_{U_{L}}^{\dagger} m_{Q}^{2} V_{U_{L}}, \quad \widetilde{m}_{D_{L}}^{2}=V_{D_{L}}^{\dagger} m_{Q}^{2} V_{D_{L}}, \quad \widetilde{m}_{U_{R}}^{2}=V_{U_{R}}^{\dagger} m_{U}^{2} V_{U_{R}}, \\
& \widetilde{m}_{D_{R}}^{2}=V_{D_{R}}^{\dagger} m_{D}^{2} V_{D_{R}}, \quad \widetilde{m}_{E_{L}}^{2}=\widetilde{m}_{\nu_{L}}^{2}=V_{E_{L}}^{\dagger} m_{L}^{2} V_{E_{L}}, \quad \widetilde{m}_{E_{R}}^{2}=V_{E_{R}}^{\dagger} m_{E}^{2} V_{E_{R}}, \\
& y_{U}^{A}=V_{U_{R}}^{\dagger} Y_{U}^{A} V_{U_{L}}, \quad y_{D}^{A}=V_{D_{R}}^{\dagger} Y_{D}^{A} V_{D_{L}}, \quad y_{E}^{A}=V_{E_{R}}^{\dagger} Y_{E}^{A} V_{E_{L}} .
\end{aligned}
$$

Trilinear terms are transformed similar to the Yukawa couplings. The left-handed neutrinos in this base are called as interaction eigenstate neutrinos. Together with the D-term contributions after the electroweak symmetry breaking, $\widetilde{m}^{2}$ and $y^{A}$ give the mass matrices of sfermions, Eq. (A.9), relevant to the low energy phenomenology. The entries in Eq. (A.9) are

$$
\begin{aligned}
& \widetilde{M}_{U_{L L}}^{2}=\widetilde{m}_{U_{L}}^{2}+m_{U}^{2}+M_{Z}^{2} \cos 2 \beta\left(\frac{1}{2}-\frac{2}{3} \sin ^{2} \theta_{W}\right), \\
& \widetilde{M}_{U_{R R}}^{2}=\widetilde{m}_{U_{R}}^{2}+m_{U}^{2}+M_{Z}^{2} \cos 2 \beta\left(\frac{2}{3} \sin ^{2} \theta_{W}\right), \\
& \widetilde{M}_{U_{L R}}^{2}=-v_{2}\left(y_{U}^{A \dagger}+\mu \cot \beta y_{U}\right) ; \\
& \widetilde{M}_{D_{L L}}^{2}=\widetilde{m}_{D_{L}}^{2}+m_{D}^{2}-M_{Z}^{2} \cos 2 \beta\left(\frac{1}{2}-\frac{1}{3} \sin ^{2} \theta_{W}\right), \\
& \widetilde{M}_{D_{R R}}^{2}=\widetilde{m}_{D_{R}}^{2}+m_{D}^{2}-M_{Z}^{2} \cos 2 \beta\left(\frac{1}{3} \sin ^{2} \theta_{W}\right), \\
& \widetilde{M}_{D_{L R}}^{2}=-v_{1}\left(y_{D}^{A \dagger}+\mu \tan \beta y_{D}\right) ; \\
& \widetilde{M}_{E_{L L}}^{2}=\widetilde{m}_{E_{L}}^{2}+m_{E}^{2}-M_{Z}^{2} \cos 2 \beta\left(\frac{1}{2}-\sin ^{2} \theta_{W}\right), \\
& \widetilde{M}_{E_{R R}}^{2}=\widetilde{m}_{E_{R}}^{2}+m_{E}^{2}-M_{Z}^{2} \cos 2 \beta \sin ^{2} \theta_{W}, \\
& \widetilde{M}_{E_{L R}}^{2}=-v_{1}\left(y_{E}^{A \dagger}+\mu \tan \beta y_{E}\right) .
\end{aligned}
$$

$m_{U}, m_{D}$ and $m_{E}$ are the diagonal SM fermion mass matrices.

Assuming the SUSY breaking is universal at $M_{G U T}$ scale, we are able to get the radiatively corrected SUSY breaking soft terms. At the first order, the radiative corrections given by the Yukawa and trilinear couplings are

$$
\delta_{1} \widetilde{m}_{U_{R}}^{2}=-\frac{2}{16 \pi^{2}}\left(3 m_{0}^{2}+\left|A_{0}\right|^{2}\right) y_{U}^{2} \Delta_{1} t
$$




$$
\begin{aligned}
\delta_{1}{\widetilde{m}_{D_{R}}^{2}}^{2} & =-\frac{1}{16 \pi^{2}}\left(3 m_{0}^{2}+\left|A_{0}\right|^{2}\right) y_{D}^{2} \Delta_{1} t, \\
\delta_{1}{\widetilde{m}_{U_{L}}^{2}}^{2} & =-\frac{1}{16 \pi^{2}}\left(3 m_{0}^{2}+\left|A_{0}\right|^{2}\right)\left(y_{U}^{2}+K y_{D}^{2} K^{\dagger}\right) \Delta_{1} t, \\
\delta_{1}{\widetilde{m}_{D_{L}}^{2}}^{2} & =-\frac{1}{16 \pi^{2}}\left(3 m_{0}^{2}+\left|A_{0}\right|^{2}\right)\left(K^{\dagger} y_{U}^{2} K+y_{D}^{2}\right) \Delta_{1} t, \\
\delta_{1}{\widetilde{m}_{E_{R}}^{2}}^{2} & =-\frac{2}{16 \pi^{2}}\left(3 m_{0}^{2}+\left|A_{0}\right|^{2}\right) y_{E}^{2} \Delta_{1} t, \\
\delta_{1} \widetilde{m}_{E_{L}}^{2} & =-\frac{1}{16 \pi^{2}}\left(3 m_{0}^{2}+\left|A_{0}\right|^{2}\right)\left(y_{E}^{2} \Delta_{1} t+U_{N}^{\dagger} y_{N}^{2} U_{N} \bar{\Delta}_{1} t\right)=\delta_{1} \widetilde{m}_{\nu_{L}}^{2}, \\
\delta_{1} y_{U}^{A} & =-\frac{3}{32 \pi^{2}} A_{0}\left[\left[3 y_{U}\left(y_{U}^{2}+\operatorname{tr}\left(y_{U}^{2}\right)\right)+y_{U} K y_{D}^{2} K^{\dagger}\right] \Delta_{1} t+y_{U} \operatorname{tr}\left(y_{N}^{2}\right) \bar{\Delta}_{1} t\right], \\
\delta_{1} y_{D}^{A} & =-\frac{3}{32 \pi^{2}} A_{0}\left[3 y_{D}\left(y_{D}^{2}+\operatorname{tr}\left(y_{D}^{2}\right)\right)+y_{D} K^{\dagger} y_{U}^{2} K+y_{D} \operatorname{tr}\left(y_{E}^{2}\right)\right] \Delta_{1} t, \\
\delta_{1} y_{E}^{A} & =-\frac{3}{32 \pi^{2}} A_{0}\left[\left[y_{E}^{3}+y_{E} \operatorname{tr}\left(y_{E}^{2}\right)+3 y_{E} \operatorname{tr}\left(y_{D}^{2}\right)\right] \Delta_{1} t+y_{E} U_{N}^{\dagger} y_{N}^{2} U_{N} \bar{\Delta}_{1} t\right], \\
\Delta_{1} t & =\ln \frac{M_{G U T}^{2}}{M_{S U S Y}^{2}}, \quad \bar{\Delta}_{1} t=\ln \frac{M_{G U T}^{2}}{M_{N}^{2}},
\end{aligned}
$$

where we assume the right-handed neutrino fields $N_{i}$ decouple at the same scale. The above estimates are presented at the first order. A detailed analysis beyond the first order has been presented in [47] for MSSM. The results are in agreement with the qualitative expectations based on the first order approximation. In the model with the right-handed neutrino fields, similar conclusion can be obtained.

\section{Appendix C: RGEs in the Flipped $S U(5)$ Model}

In the flipped $S U(5)$ model, the RGEs are obtained as follows

$$
\begin{aligned}
\frac{d \tilde{Y}_{10}}{d t}= & \left(2 C_{10}+C_{h}\right) \tilde{\alpha}_{5} \tilde{Y}_{10}+\left(2 C_{10}^{X}+C_{h}^{X}\right) \tilde{\alpha}_{X} \tilde{Y}_{10}-3 \tilde{Y}_{10} \tilde{Y}_{10}^{\dagger} \tilde{Y}_{10} \\
& -\left[\tilde{Y}_{\overline{5}} \tilde{Y}_{\overline{5}}^{\dagger} \tilde{Y}_{10}+\tilde{Y}_{10}\left(\tilde{Y}_{\overline{5}} \tilde{Y}_{\overline{5}}^{\dagger}\right)^{T}\right]-\frac{1}{2}\left[3 \operatorname{tr}\left(\tilde{Y}_{10} \tilde{Y}_{10}^{\dagger}\right)+\operatorname{tr}\left(\tilde{Y}_{1} \tilde{Y}_{1}^{\dagger}\right)\right] \tilde{Y}_{10} \\
\frac{d \tilde{Y}_{\overline{5}}}{d t}= & \left(C_{10}+C_{5}+C_{h}\right) \tilde{\alpha}_{5} \tilde{Y}_{\overline{5}}+\left(C_{10}^{X}+C_{5}^{X}+C_{h}^{X}\right) \tilde{\alpha}_{X} \tilde{Y}_{\overline{5}} \\
& -\frac{3}{2} \tilde{Y}_{10} \tilde{Y}_{10}^{\dagger} \tilde{Y}_{\overline{5}}-3 \tilde{Y}_{\overline{5}} \tilde{Y}_{\overline{5}}^{\dagger} \tilde{Y}_{\overline{5}}-\frac{1}{2} \tilde{Y}_{\overline{5}} \tilde{Y}_{1}^{\dagger} \tilde{Y}_{1}-2 \operatorname{tr}\left(\tilde{Y}_{\overline{5}} \tilde{Y}_{\overline{5}}^{\dagger}\right) \tilde{Y}_{\overline{5}} \\
\frac{d \tilde{Y}_{1}}{d t}= & \left(C_{5}+C_{h}\right) \tilde{\alpha}_{5} \tilde{Y}_{1}+\left(C_{5}^{X}+C_{h}^{X}+C_{1}^{X}\right) \tilde{\alpha}_{X} \tilde{Y}_{1}
\end{aligned}
$$




$$
-3 \tilde{Y}_{1} \tilde{Y}_{1}^{\dagger} \tilde{Y}_{1}-2 \tilde{Y}_{1} \tilde{Y}_{\overline{5}}^{\dagger} \tilde{Y}_{\overline{5}}-\frac{1}{2}\left[\operatorname{tr}\left(\tilde{Y}_{1} \tilde{Y}_{1}^{\dagger}\right)+3 \operatorname{tr}\left(\tilde{Y}_{10} \tilde{Y}_{10}\right)\right] \tilde{Y}_{1} .
$$

Here we have $t=\ln \frac{M_{*}^{2}}{Q^{2}}$ and the following Casimir operators:

$$
C_{10}=\frac{18}{5}, C_{h}=C_{5}=\frac{12}{5}, C_{10}^{X}=\frac{1}{40}, C_{5}^{X}=\frac{9}{40}, C_{1}^{X}=\frac{5}{8}, C_{h}^{X}=\frac{1}{10} .
$$

$\alpha_{5}$ and $\alpha_{X}$ are the gauge couplings of $S U(5)$ and $U(1)_{X}$ gauge groups, respectively. For the SUSY breaking soft terms, we have

$$
\begin{aligned}
\frac{d \tilde{Y}_{10}^{A}}{d t}= & \left(2 C_{10}+C_{h}\right) \tilde{\alpha}_{5}\left(\tilde{Y}_{10}^{A}+2 M_{5} \tilde{Y}_{10}\right)+\left(2 C_{10}^{X}+C_{h}^{X}\right) \tilde{\alpha}_{X}\left(\tilde{Y}_{10}^{A}+2 M_{X} \tilde{Y}_{10}\right) \\
& -\frac{9}{2}\left(\tilde{Y}_{10}^{A} \tilde{Y}_{10}^{\dagger} \tilde{Y}_{10}+\tilde{Y}_{10} \tilde{Y}_{10}^{\dagger} \tilde{Y}_{10}^{A}\right)-2\left[\tilde{Y}_{\overline{5}}^{A} \tilde{Y}_{\overline{5}}^{\dagger} \tilde{Y}_{10}+\tilde{Y}_{10}\left(\tilde{Y}_{\overline{5}}^{A} \tilde{Y}_{\overline{5}}^{\dagger}\right)^{T}\right]-\left[\tilde{Y}_{\overline{5}} \tilde{Y}_{\overline{5}}^{\dagger} \tilde{Y}_{10}^{A}+\tilde{Y}_{10}^{A}\left(\tilde{Y}_{\overline{5}} \tilde{Y}_{\overline{5}}^{\dagger}\right)^{T}\right] \\
& -\frac{3}{2}\left[2 \operatorname{tr}\left(\tilde{Y}_{10}^{A} \tilde{Y}_{10}^{\dagger}\right) \tilde{Y}_{10}+\operatorname{tr}\left(\tilde{Y}_{10} \tilde{Y}_{10}^{\dagger}\right) \tilde{Y}_{10}^{A}\right]-\frac{1}{2}\left[2 \operatorname{tr}\left(\tilde{Y}_{1}^{A} \tilde{Y}_{1}^{\dagger}\right) \tilde{Y}_{10}+\operatorname{tr}\left(\tilde{Y}_{1} \tilde{Y}_{1}^{\dagger}\right) \tilde{Y}_{10}^{A}\right] \\
\frac{d \tilde{Y}_{5}^{A}}{d t}= & \left(C_{10}+C_{5}+C_{h}\right) \tilde{\alpha}_{5}\left(\tilde{Y}_{5}^{A}+2 M_{5} \tilde{Y}_{\overline{5}}\right)+\left(C_{10}^{X}+C_{5}^{X}+C_{h}^{X}\right) \tilde{\alpha}_{X}\left(\tilde{Y}_{5}^{A}+2 M_{X} \tilde{Y}_{5}\right) \\
& -\frac{3}{2} \tilde{Y}_{10} \tilde{Y}_{10}^{\dagger} \tilde{Y}_{5}^{A}-3 \tilde{Y}_{10}^{A} \tilde{Y}_{10}^{\dagger} \tilde{Y}_{\overline{5}}-4 \tilde{Y}_{5}^{A} \tilde{Y}_{\overline{5}}^{\dagger} \tilde{Y}_{\overline{5}}-5 \tilde{Y}_{\overline{5}} \tilde{Y}_{5}^{\dagger} \tilde{Y}_{5}^{A} \\
& -\frac{1}{2} \tilde{Y}_{\overline{5}}^{A} \tilde{Y}_{1}^{\dagger} \tilde{Y}_{1}-\tilde{Y}_{5} \tilde{Y}_{1}^{\dagger} \tilde{Y}_{1}^{A}-2 \operatorname{tr}\left(\tilde{Y}_{5} \tilde{Y}_{5}^{\dagger}\right) \tilde{Y}_{\overline{5}}^{A}-4 \operatorname{tr}\left(\tilde{Y}_{5}^{A} \tilde{Y}_{5}^{\dagger}\right) \tilde{Y}_{\overline{5}} \\
\frac{d \tilde{Y}_{1}^{A}}{d t}= & \left(C_{5}+C_{h}\right) \tilde{\alpha}_{5}\left(\tilde{Y}_{1}^{A}+2 M_{5} \tilde{Y}_{1}\right)+\left(C_{5}^{X}+C_{h}^{X}+C_{1}^{X}\right) \tilde{\alpha}_{X}\left(\tilde{Y}_{1}^{A}+2 M_{X} \tilde{Y}_{1}\right) \\
& -\frac{7}{2} \tilde{Y}_{1} \tilde{Y}_{1}^{\dagger} \tilde{Y}_{1}^{A}-\frac{11}{2} \tilde{Y}_{1}^{A} \tilde{Y}_{1}^{\dagger} \tilde{Y}_{1}-2 \tilde{Y}_{1}^{A} \tilde{Y}_{\overline{5}}^{\dagger} \tilde{Y}_{\overline{5}}-4 \tilde{Y}_{1} \tilde{Y}_{5}^{\dagger} \tilde{Y}_{\overline{5}}^{A} \\
& -\frac{1}{2}\left[\operatorname{tr}\left(\tilde{Y}_{1} \tilde{Y}_{1}^{\dagger}\right) \tilde{Y}_{1}^{A}+2 \operatorname{tr}\left(\tilde{Y}_{1}^{A} \tilde{Y}_{1}^{\dagger}\right) \tilde{Y}_{1}\right]-\frac{3}{2}\left[\operatorname{tr}\left(\tilde{Y}_{10} \tilde{Y}_{10}^{\dagger}\right) \tilde{Y}_{1}^{A}+2 \operatorname{tr}\left(\tilde{Y}_{10}^{A} \tilde{Y}_{10}^{\dagger}\right) \tilde{Y}_{1}\right]
\end{aligned}
$$

$$
\begin{aligned}
\frac{d m_{10}^{2}}{d t}= & 4 C_{10} \tilde{\alpha}_{5}\left|M_{5}\right|^{2}+4 C_{10}^{X} \tilde{\alpha}_{X}\left|M_{X}\right|^{2}-\frac{3}{2}\left[m_{10}^{2}\left(\tilde{Y}_{10} \tilde{Y}_{10}^{\dagger}\right)^{T}+\tilde{Y}_{10} \tilde{Y}_{10}^{\dagger} m_{10}^{2}\right] \\
& -\left[m_{10}^{2}\left(\tilde{Y}_{\overline{5}} \tilde{Y}_{\overline{5}}^{\dagger}\right)^{T}+\left(\tilde{Y}_{\overline{5}} \tilde{Y}_{\overline{5}}^{\dagger}\right)^{T} m_{10}^{2}\right]-3\left(\tilde{Y}_{10}^{A} \tilde{Y}_{10}^{A \dagger}+\tilde{Y}_{10} m_{10}^{2} \tilde{Y}_{10}^{\dagger}\right)^{T} \\
& -2\left(\tilde{Y}_{\overline{5}}^{A} \tilde{Y}_{\overline{5}}^{A \dagger}+\tilde{Y}_{\overline{5}} m_{\overline{5}}^{2} \tilde{Y}_{\overline{5}}^{\dagger}\right)^{T}-3\left(\tilde{Y}_{10} \tilde{Y}_{10}^{\dagger}\right)^{T} m_{h}^{2}-2\left(\tilde{Y}_{\overline{5}} \tilde{Y}_{\overline{5}}^{\dagger}\right)^{T} m_{\bar{h}}^{2}
\end{aligned}
$$$$
\frac{d m_{5}^{2}}{d t}=4 C_{5} \tilde{\alpha}_{5}\left|M_{5}\right|^{2}+4 C_{5}^{X} \tilde{\alpha}_{X}\left|M_{X}\right|^{2}-2\left(m_{5}^{2} \tilde{Y}_{\overline{5}}^{\dagger} \tilde{Y}_{\overline{5}}+\tilde{Y}_{5}^{\dagger} \tilde{Y}_{5} m_{5}^{2}\right)-\frac{1}{2}\left(m_{5}^{2} \tilde{Y}_{1}^{\dagger} \tilde{Y}_{1}+\tilde{Y}_{1}^{\dagger} \tilde{Y}_{1} m_{5}^{2}\right)
$$$$
-4\left(\tilde{Y}_{\overline{5}}^{\dagger}\left(m_{10}^{2}\right)^{T} \tilde{Y}_{\overline{5}}+\tilde{Y}_{\overline{5}}^{A \dagger} \tilde{Y}_{\overline{5}}^{A}\right)-\left(\tilde{Y}_{1}^{\dagger}\left(m_{1}^{2}\right)^{T} \tilde{Y}_{1}+\tilde{Y}_{1}^{A \dagger} \tilde{Y}_{1}^{A}\right)-4 \tilde{Y}_{\overline{5}}^{\dagger} \tilde{Y}_{\overline{5}} m_{\bar{h}}^{2}-\tilde{Y}_{1}^{\dagger} \tilde{Y}_{1} m_{h}^{2},
$$

$$
\frac{d m_{1}^{2}}{d t}=4 \tilde{\alpha}_{X} C_{1}^{X}\left|M_{X}\right|^{2}-\frac{5}{2}\left(\left(\tilde{Y}_{1} \tilde{Y}_{1}^{\dagger}\right)^{T} m_{1}^{2}+m_{1}^{2}\left(\tilde{Y}_{1} \tilde{Y}_{1}^{\dagger}\right)^{T}\right)
$$




$$
\begin{aligned}
& -5\left(\tilde{Y}_{1} m_{\overline{5}}^{2} \tilde{Y}_{1}^{\dagger}+\tilde{Y}_{1}^{A} \tilde{Y}_{1}^{A \dagger}\right)^{T}-5\left(\tilde{Y}_{1} \tilde{Y}_{1}^{\dagger}\right)^{T} m_{h}^{2} \\
\frac{d m_{h}^{2}}{d t}= & 4 C_{h} \tilde{\alpha}_{5}\left|M_{5}\right|^{2}+4 C_{h}^{X} \tilde{\alpha}_{X}\left|M_{X}\right|^{2}-3 \operatorname{tr}\left[\tilde{Y}_{10} \tilde{Y}_{10}^{\dagger} m_{h}^{2}+2 \tilde{Y}_{10} m_{10}^{2} \tilde{Y}_{10}^{\dagger}+\tilde{Y}_{10}^{A} \tilde{Y}_{10}^{A \dagger}\right] \\
& -\operatorname{tr}\left[\tilde{Y}_{1} \tilde{Y}_{1}^{\dagger} m_{h}^{2}+\tilde{Y}_{1} m_{\overline{5}}^{2} \tilde{Y}_{1}^{\dagger}+\tilde{Y}_{1}^{T} m_{1}^{2} \tilde{Y}_{1}^{*}+\tilde{Y}_{1}^{A} \tilde{Y}_{1}^{A \dagger}\right] \\
\frac{d m_{\bar{h}}^{2}}{d t}= & 4 C_{h} \tilde{\alpha}_{5}\left|M_{5}\right|^{2}+4 C_{h}^{X} \tilde{\alpha}_{X}\left|M_{X}\right|^{2}-4 \operatorname{tr}\left[\tilde{Y}_{\overline{5}} \tilde{Y}_{\overline{5}}^{\dagger} m_{\bar{h}}^{2}+\tilde{Y}_{\overline{5}} m_{\overline{5}}^{2} \tilde{Y}_{\overline{5}}^{\dagger}+\tilde{Y}_{\overline{5}}^{T} m_{10}^{2} \tilde{Y}_{\overline{5}}^{*}+\tilde{Y}_{\overline{5}}^{A} \tilde{Y}_{\overline{5}}^{A \dagger}\right]
\end{aligned}
$$

According to the above RGEs, we can estimate the first order radiative corrections to the SUSY breaking soft terms. Using Eqs. (B.17), (13) and (32), we can write them in the superCKM base and see clearly the extra flavor structure. Assuming the universal SUSY breaking soft terms, $m_{0}$ for scalars, $M_{\frac{1}{2}}$ for gauginos and $Y^{A}=A_{0} Y$ at the $M_{*}$ scale, we get the following corrections given by the Yukawa and trilinear couplings

$$
\begin{aligned}
\delta_{2} \widetilde{m}_{D_{L}}^{2}= & -\frac{1}{16 \pi^{2}}\left[3\left(3 m_{0}^{2}+\left|A_{0}\right|^{2}\right) y_{D}^{2}+2\left(3 m_{0}^{2}+\left|A_{0}\right|^{2}\right) K^{\dagger} y_{U}^{2} K\right] \Delta_{2} t \\
\delta_{2} \widetilde{m}_{U_{L}}^{2}= & -\frac{1}{16 \pi^{2}}\left[3\left(3 m_{0}^{2}+\left|A_{0}\right|^{2}\right) K y_{D}^{2} K^{\dagger}+2\left(3 m_{0}^{2}+\left|A_{0}\right|^{2}\right) y_{U}^{2}\right] \Delta_{2} t \\
\delta_{2} \widetilde{m}_{U_{R}}^{2}= & -\frac{1}{16 \pi^{2}}\left[4\left(3 m_{0}^{2}+\left|A_{0}\right|^{2}\right) y_{U}^{2}+\left(3 m_{0}^{2}+\left|A_{0}\right|^{2}\right) U_{N}^{*} y_{E}^{2} U_{N}^{T}\right] \Delta_{2} t \\
\delta_{2} \widetilde{m}_{D_{R}}^{2}= & \left.-\frac{1}{16 \pi^{2}}\left[3\left(3 m_{0}^{2}+\left|A_{0}\right|^{2}\right) y_{D}^{2}+2\left(3 m_{0}^{2}+\left|A_{0}\right|^{2}\right) K^{T} y_{U}^{2} K^{*}\right)\right] \Delta_{2} t \\
\delta_{2} \widetilde{m}_{E_{L}}^{2}= & -\frac{1}{16 \pi^{2}}\left[\left(3 m_{0}^{2}+\left|A_{0}\right|^{2}\right) y_{E}^{2}+4\left(3 m_{0}^{2}+\left|A_{0}\right|^{2}\right) U_{N}^{\dagger} y_{U}^{2} U_{N}\right] \Delta_{2} t \\
\delta_{2} \widetilde{m}_{E_{R}}^{2}= & -\frac{1}{16 \pi^{2}} 5\left(3 m_{0}^{2}+\left|A_{0}\right|^{2}\right) y_{E}^{2} \Delta_{2} t \\
\delta_{2} y_{U}^{A}= & -\frac{A_{0}}{16 \pi^{2}}\left[\frac{9}{2} y_{U} K y_{D}^{2} K^{\dagger}+9 y_{U}^{3}+\frac{3}{2} U_{N}^{*} y_{E}^{2} U_{N}^{T} y_{U}+6 \operatorname{tr}\left(y_{U}^{2}\right) y_{U}\right] \Delta_{2} t \\
\delta_{2} y_{D}^{A}= & -\frac{A_{0}}{16 \pi^{2}}\left[9 y_{D}^{3}+3\left(y_{D} K^{\dagger} y_{U}^{2} K+K^{T} y_{U}^{2} K^{*} y_{D}\right)+\frac{3}{2}\left(\operatorname{tr}\left(y_{E}^{2}\right)+3 \operatorname{tr}\left(y_{D}^{2}\right)\right) y_{D}\right] \Delta_{2} t,(\mathrm{C} .2 \\
\delta_{2} y_{E}^{A}= & -\frac{A_{0}}{16 \pi^{2}}\left[9 y_{E}^{3}+5 y_{E} U_{N}^{\dagger} y_{U}^{2} U_{N}+\frac{3}{2}\left(\operatorname{tr}\left(y_{E}^{2}\right)+3 \operatorname{tr}\left(y_{D}^{2}\right)\right) y_{E}\right] \Delta_{2} t
\end{aligned}
$$

\section{References}

[1] Y. Fukuda et al. [Super-Kamiokande Collaboration], Phys. Rev. Lett. 81(1998)1562.

[2] Q.R. Ahmad et al. [SNO Collaboration], Phys. Rev. Lett. 89(2002)011301; Phys. Rev. Lett. 89(2002)011302. 
[3] K. Eguchi et al. [KamLAND Collaboration], Phys. Rev. Lett. 90(2003)021802.

[4] M. Apollonio et al.[CHOOZ Collaboration], Phys.Lett.B466(1999)415.

[5] M. Shiozawa, talk given at the ICHEP2002 for the Super-K collaboration;

[6] P.C. de Holanda and A.Yu. Smirnov, hep-ph/0212270.

[7] see also M. Maltoni, T. Schwetz, M.A. Tortola, J.W.F. Valle, Phys. Rev. D67(2003)013011[hep-ph/0207227]; S. Pakvasa, J. W. F. Valle, hep-ph/0301061.

[8] B. Pontecorva, Sov. Phys. JETP 6(1958)429.

[9] Z. Maki, M. Nakagawa and S. Sakata, Prog. Theor. Phys. 28, 870 (1962).

[10] L. Wolfenstein, Phys. Rev. D17(1978)2369; S.P. Mikheev and A.Yu. Smirnov, Sov. J. Nucl. Phys. 42(1985)913, Yad. Fiz. 42(1985)1441.

[11] T. Yanagida, in Proc. of the Workshop on the Unified Theory and Baryon Number in the Universe, ed. O. Sawada and A. Sugamoto (KEK report 79-18, 1979), p. 95;

M. Gell-Mann, P. Ramond and R. Slansky, in Supergravity, ed. P. van Nieuwenhuizen and D.Z. Freedman (North Holland, Amsterdam, 1979), p. 315; E. Witten, Phys. Lett. B 91, 81 (1980); R. N. Mohapatra and G. Senjanovic, Phys. Rev. Lett. 44, 912 (1980);

T. Yanagida, Prog. Theor. Phys. 64, 1103 (1980).

[12] S. Weinberg, Trans.N.Y.Acad.Sci.38(1977)185; F. Wilczek and A. Zee, Phys. Lett. B70(1977)418; H. Fritzsch, Phys. Lett. B70(1977)436.

[13] C. D. Froggatt and H. B. Nielsen, Nucl. Phys. B147 (1979) 277.

[14] K.S. Babu and S.M. Barr, Phys. Lett. B 381(1996) 202; C.H. Albright, K.S. Babu, and S.M. Barr, Phys. Rev. Lett. 81(1998)1167; J. Sato and T. Yanagida, Phys. Lett. B 430(1998)127; N. Irges, S. Lavignac, and P. Ramond, Phys. Rev. D 581998)035003.

[15] W. Buchmuller, D. Delepine and F. Vissani, Phys. Lett. B459 171 (1999); W. Buchmuller, D. Delepine and L. T. Handoko, Nucl. Phys. B576 445 (2000); J. Ellis, M. E. Gomez, G. K. Leontaris, S. Lola and D. V. Nanopoulos, Eur. Phys. J. C14 319 (2000); J. Hisano and K. Tobe, Phys. Lett. B510 197 (2001); J. A. Casas and A. Ibarra, arXiv:hep-ph/0103065; D. F. Carvalho, J. Ellis, M. E. Gomez and S. Lola, arXiv:hep-ph/0103256; T. Blazek and S. F. King, arXiv:hep-ph/0105005; J. Sato, K. Tobe and T. Yanagida, Phys. Lett. B498 189 (2001); J. Sato and K. Tobe, Phys. Rev. D63 116010 (2001); S. Lavignac, I. Masina and C. A. Savoy, arXiv:hep-ph/0106245; T. Moroi, JHEP. 0003019 (2000), [arXiv:hepph/0002208]; N. Akama, Y. Kiyo, S. Komine and T. Moroi, Phys. Rev. D64 095012 (2001) [arXiv:hep-ph/0104263]; T. Moroi, Phys. Lett. B493, 366 (2000) [arXiv: hep-ph/0007328].

[16] J. Hisano, T. Moroi, K. Tobe and M. Yamaguchi, Phys. Rev. D53(1996)2442[hepph/9510309]; J. Hisano and D. Nomura, Phys. Rev. D59(1999)116005[hep-ph/9810479].

[17] D. Chang, A. Masiero and H. Murayama, hep-ph/0205111.

[18] A. Masiero, S. K. Vempati and O. Vives, Nucl. Phys. B649(2003)189[hep-ph/0209303]. 
[19] B. Bajc, G. Senjanović and F. Vissani, hep-ph/0210207; H.S. Goh, R.N. Mohapatra and S.-P. Ng, hep-ph/0303055.

[20] J. Hisano and Y. Shimizu, hep-ph/0303071.

[21] F. Borzumati and A. Masiero, Phys. Rev. Lett. 57(1986)961.

[22] R. Barbieri and L.J. Hall, Phys. Lett. B338(1994)212; R. Barbieri, L.J. Hall and A. Strumia, Nucl. Phys. B445(1995)219.

[23] L.J. Hall, V.A. Kostelecky and S. Raby, Nucl. Phys. B267 (1986)415.

[24] J. Ellis, J.L. Lopez and D.V. Nanopoulos, Phys. Lett. B245 (1990) 375; A. Font, L. Ibáñez and F. Quevedo, Nucl. Phys. B345 (1990) 389.

[25] A. E. Faraggi, D. V. Nanopoulos and K. j. Yuan, Nucl. Phys. B 335, 347 (1990); A. E. Faraggi, Nucl. Phys. B 387, 239 (1992), and references therein.

[26] I. Antoniadis, G. K. Leontaris and J. Rizos, Phys. Lett. B 245, 161 (1990).

[27] I. Antoniadis, J. R. Ellis, J. S. Hagelin and D. V. Nanopoulos, Phys. Lett. B 231, 65 (1989); J. L. Lopez, D. V. Nanopoulos and K. j. Yuan, Nucl. Phys. B 399, 654 (1993), and references therein.

[28] M. Duncan, Nucl. Phys. B221(1983)285; J. Donoghue, H. Nilles and D. Wyler, Phys. Lett. B128(1983)55; A. Bouquet, J. Kaplan and C. Savoy, Phys. Lett. B148(1984)69.

[29] S. M. Barr, Phys. Lett. B 112, 219 (1982); see also A. De Rujula, H. Georgi and S. L. Glashow, Phys. Rev. Lett. 45, 413 (1980).

[30] J. P. Derendinger, J. E. Kim and D. V. Nanopoulos, Phys. Lett. B 139, 170 (1984); I. Antoniadis, J. R. Ellis, J. S. Hagelin and D. V. Nanopoulos, Phys. Lett. B 194, 231 (1987).

[31] H. Fusaoka and Y. Koide, Phys. Rev. D 57, 3986 (1998).

[32] P.H. Chankowski , W. Krolikowski and S. Pokorski, Phys.Lett. B473(2000)109[hepph/9910231] and references therein.

[33] F. Gabbiani, E. Garieli, A. Masiero and L. Silvestrini, Nucl.Phys. B477(1996)321[hep$\mathrm{ph} / 9604387]$.

[34] M. Misiak, S. Pokorski and J. Rosiek, in Heavy Flavours II, eds. A.J. Buras and M. Lindner, Advanced Series on Directions in High Energy Physics, World Scientific 1997[hepph/9703442].

[35] A.J. Buras, P.H. Chankowski, J. Rosiek and L. Slawianowska, hep-ph/0210145.

[36] S. L. Glashow, J. Iliopoulos, and L. Maiani, Phys. Rev. D2(1970)1285.

[37] K. Hagiwara et.al, Review of the Particle Physics, Phys.RevD66(2002)010001. 
[38] D. Chang, W-F. Chang, W-Y. Keung, N. Sinha and R. Sinha, Phys. Rev. D65(2002)055010[hep-ph/0109151].

[39] J. Edsjö, hep-ph/0301106; H. Baer and C. Balazs, hep-ph/0303114; A.B. Lahanas and D.V. Nanopoulos, hep-ph/0303130.

[40] J. Hisano, T. Moroi, K. Tobe and M. Yamaguchi, Phys.Lett. B391(1997)341[hepph/9605296]; (E)ibid. B397(1997)357.

[41] B. Mele, S. Petrarca and A. Soddu, Phys. Lett. B435 (1998) 401; G. Eilam, J.L. Hewett and A. Soni, Phys. Rev. D44 (1991) 1473, Erratum: Phys. Rev. D59 (1998) 039901.

[42] W.S. Hou, Phys. Lett. B296 (1992) 179; K. Agashe and M. Grzaesser, Phys. Rev. D54 (1996) 4445; M. Hosch, K. Whisnant and B.L. Young, Phys. Rev. D56 (1997) 5725.

[43] J. Guasch and J. Solá, hep-ph/9906268. For earier liturature, C.S. Li, R.J. Oakes, J.M. Yang, Phys. Rev. D49 (1994) 293, Erratum: ibid. D56 (1997) 3156; J.M. Yang and C.S. Li, Phys. Rev. D49 (1994) 3412; G. Couture, C. Hamzaoui, H. König, Phys. Rev. D52 (1995) 1713; G. Couture, M. Frank, H. König, Phys. Rev. D56 (1997) 4213; J.L. Lopez, D.V. Nanopoulos, R. Rangarajan, Phys. Rev. D56 (1997) 3100; G.M. de Divitiis, R. Petronzio, L. Silverstini, Nucl. Phys. B504 (1997) 45; J.M. Yang, B. Young and X. Zhang, Phys. Rev. D58 (1998) 055001; G. Eilam et al., Phys. Lett. B510 (2001) 227.

[44] Proceedings of the Workshop on Standard Model Physics (and More) at the LHC, ed. G. Altarelli and M.L. Mangono, CERN 2000-004.

[45] J. Cao, Z. Xiong and J.-M. Yang, hep-ph/0208035; C.-S. Huang, X.-H. Wu and S.-H. Zhu, Phys. Lett. B452 (1999) 143; T. Han, J. Hewett, hep-ph/9811237; U. Mahanta, A. Ghosal, Phys. Rev. D57 (1998) 1735; Y. Koide, hep-ph/9701261; D. Atwood, L. Reina, A. Soni, Phys. Rev. D53 (1996) 1199. For the earier liturature, see, for example, the references in Phys. Lett. B452 (1999) 143 and hep-ph/0208035.

[46] S. Dimopoulos, L.J. Hall, Phys. Lett. B344(1995)185; R. Barbieri, L.J. Hall and A. Strumia, Nucl. Phys. B449(1995)437; Phys. Lett. B369(1996)283.

[47] N. Arkani-Hamed, Hsin-Chia Cheng and L.J. Hall, Phys. Rev. D53(1996)413. 